\title{
Which Random Walk is Faster? Methods to Compare Different Step Length Distributions in Individual Animal Movement
}

\author{
J. D. Choules, S. Petrovskii * \\ Department of Mathematics, University of Leicester, Leicester, LE1 7RH, UK
}

\begin{abstract}
Good understanding of individual animal movement is needed in the context of epidemiology in order to predict the rate of spread of infectious diseases. It is also required for problems arising in nature conservation, biological invasion, pest monitoring, etc. A question that often appears in the centre of the movement studies is which movement pattern is 'faster' or more efficient. For instance, it is widely believed that the pattern quantified by a power law distribution of movement steps is faster than the Brownian motion. Here we show that the answer to this question may be not so straightforward and depends on the way how different step length distributions are compared.
\end{abstract}

Keywords and phrases: random walks, probability distributions, individual animal movement

Mathematics Subject Classification: 60G50

\section{Introduction}

Recent decades have seen outbreaks of several dangerous infectious diseases across the world such as Swine influenza [1,2], Ebola fever [3], dengue fever [4,5], Zika fever [6] and others. Once a disease outbreak occurs, one immediate task is to localize it in space, i.e. to block its spatial spread or, in case blocking is not possible, at least to predict the direction and rate of the spread. The latter is important as it provides an estimate of the time that is available to local authorities and medical units to mobilize their resources. On a global scale, it also contributes to creating an efficient framework for epidemics forecast and control [7]. Therefore, there is a need for a good understanding of the factors controlling the rate of disease spatial spread, which is a timely problem of high practical importance. Factors controlling the disease spread depend on the modes of its transmission. In the case of the human-to-human transmission, peculiarities of human travel are most important [8], including the properties of the relevant transport networks [9]. Alternatively, many diseases are vector-borne, e.g. transmitted by insects; examples are given by malaria, dengue fever and Zika virus. Good understanding of the rates and patterns of insect movement is therefore necessary, and this is one of the reasons why individual animal movement (including insects) has been a focus of intense research and controversy over the last few decades [10-13]. A question that has often

\footnotetext{
${ }^{*}$ Corresponding author. E-mail: jdc39@le.ac.uk
} 
appeared in the focus of the movement studies is which movement pattern is 'faster' - or, ultimately, more efficient, e.g. that allows a foraging animal to find its food item faster or more food items over the same time. For instance, it has been a consensus that Lévy walks are 'faster' than Brownian motion. In this paper, however, we revisit this problem and show that the answer in fact is not obvious and depends on the way how different movement patterns are compared.

Mathematical modeling of the individual movement of 'particles', whether they are animals, seeds, pollen or bacteria, has long been a major focus in ecology and biology using a variety of stochastic (e.g. random walk) and deterministic (e.g. diffusion equation) theoretical approaches $[10,12,14-20]$. The choice of the probability density function for the distribution of step length (also referred to as the dispersal kernel) is usually a central part of the model. In the deterministic framework, it affects the type of the equation that describes the evolution of the probability density function to find the moving 'particle' at a given location in space, so that the standard diffusion equation may be replaced by a fractional diffusion model [17]. For the explicitly stochastic, individual-based modeling [20? -22], which is the framework that we consider in this paper, the choice of the step length distribution equates to the assumption of the movement pattern such as given, for instance, by the Brownian motion for thin-tailed (an exponentiallybounded) dispersal kernel or Lévy walks [24] for a fat-tailed one (i.e. decaying as a power law with the exponent between 1 and 3) [11]. The latter is thought to be the movement strategy actually employed by many animal species [25-27] as it is known to, under certain conditions, optimize their foraging [28].

Thus, the main theoretical argument behind the choice of a fat-tailed dispersal kernel is that the corresponding dispersing animal moves 'faster', i.e. explore the space more efficiently, whilst a thin-tailed kernel is thought to correspond to a slower, less efficient movement [28]. In this paper, however, we argue that this is not necessarily true. We consider several different methods to compare different step length distributions and show that different methods can give different results.

A generic idea behind the comparison of different step length distribution is to equate the corresponding characteristic movement length. Such a characteristic length is usually thought of as the square root of the variance of the dispersal kernel. The processes described by a fat-tailed kernel where the variance does not exist (because of the slow decay at the kernel tail) are often called scale-free [29,30]. However, this terminology is rather misleading as there are several different ways how a characteristic length can be constructed from a given kernel [31] Moreover, the approach based on equating the variance is not really illuminating as it leaves out of scope the most interesting cases of fat-tailed kernels, instead embracing only the well-known and well-understood movement patterns that are equivalent to the Brownian motion. Therefore, an alternative and less restrictive approach is required allowing us to equate dispersal kernels with different properties. However, it is not at all clear how different ways to compare the step length distribution may affect the conclusion as to which dispersal kernel corresponds to the fastest dispersal.

The distributions we have chosen to compare for this paper are a few variations on the exponential/normal distribution, i.e. the exponential family [34-36], as well as power-law based distributions. The variants of the exponential/normal distribution are similar to $e^{-a x^{\mu}}$ where $\mu$ affects the shape and tail of the distribution, how thick or thin the tail is, and $a$ relates to the variance, the normal and exponential distributions are examples of these. There are two types of power-law distributions that we will be looking at; these are distributions based on $(x+h)^{-\mu}$ and $\left(x^{\mu}+h^{\mu}\right)^{-1}$ where $\mu$ again affects the tail of the distribution and $h$ relates to the variance.

\section{Method}

The idea of our approach is as follows. We will be comparing the distributions by altering the different parameters, $\sigma, \lambda, a$ or $h$, to make a certain characteristic value or quantity, such as the variance, equal for all distributions. Once we have found the parameters that equalize the distributions (using different characteristic quantities) we will then find the mean, the square mean and the variance. These will then be used to calculate the mean squared displacement (MSD), $E\left(R_{n}^{2}\right)$, either theoretically by using the 


\begin{tabular}{|c|c|c|c|}
\hline Distributions & $\begin{array}{c}\text { Mean Distance } \\
-E(x)\end{array}$ & $\begin{array}{l}\text { Mean Squared Distance } \\
-E\left(x^{2}\right)\end{array}$ & Variance \\
\hline $\begin{array}{l}\frac{\sqrt{2}}{\sigma \sqrt{\pi}} e^{-\frac{x^{2}}{2 \sigma^{2}}} \text { or } \\
\text { Half-Normal }\end{array}$ & $\sqrt{\frac{2}{\pi}} \sigma$ & $\sigma^{2}$ & $\frac{\pi-2}{\pi} \sigma^{2}$ \\
\hline$\frac{a^{\frac{2}{3}}}{\Gamma\left(\frac{5}{3}\right)} e^{-a x^{1.5}}$ & $\frac{2 \Gamma\left(\frac{4}{3}\right)}{3 \Gamma\left(\frac{5}{3}\right)} a^{-\frac{2}{3}}$ & $\frac{2}{3 \Gamma\left(\frac{5}{3}\right)} a^{-\frac{4}{3}}$ & $\frac{6 \Gamma\left(\frac{5}{3}\right)-2\left(\Gamma\left(\frac{4}{3}\right)\right)^{2}}{9\left(\Gamma\left(\frac{5}{3}\right)\right)^{2}} a^{-\frac{4}{3}}$ \\
\hline $\begin{array}{c}\lambda e^{-\lambda x} \text { or } \\
\text { Exponential }\end{array}$ & $\lambda^{-1}$ & $2 \lambda^{-2}$ & $\lambda^{-2}$ \\
\hline$\frac{a^{2}}{2} e^{-a \sqrt{x}}$ & $6 a^{-2}$ & $120 a^{-4}$ & $84 a^{-4}$ \\
\hline$\frac{a^{3}}{6} e^{-a \sqrt[3]{x}}$ & $60 a^{-3}$ & $20160 a^{-6}$ & $16560 a^{-6}$ \\
\hline $\begin{array}{c}\frac{\mu-1}{h^{1-\mu}}(x+h)^{-\mu} \text { or } \\
\text { Power-Law }\end{array}$ & $\frac{h}{\mu-2}$ & $\frac{2 h^{2}}{(\mu-2)(\mu-3)}$ & $\frac{(\mu-1) h^{2}}{(\mu-2)^{2}(\mu-3)}$ \\
\hline$\frac{3 h^{5}}{\pi}\left(x^{6}+h^{6}\right)^{-1}$ & $\frac{h}{\sqrt{3}}$ & $\frac{h^{2}}{2}$ & $\frac{h^{2}}{6}$ \\
\hline$\frac{2^{\frac{3}{2}} h^{3}}{\pi}\left(x^{4}+h^{4}\right)^{-1}$ & $\frac{h}{\sqrt{2}}$ & $h^{2}$ & $\frac{h^{2}}{2}$ \\
\hline$\frac{3 \sqrt{3} h^{2}}{2 \pi}\left(x^{3}+h^{3}\right)^{-1}$ & $h$ & $\mathrm{~N} / \mathrm{A}$ & $\mathrm{N} / \mathrm{A}$ \\
\hline$\frac{2 h}{\pi}\left(x^{2}+h^{2}\right)^{-1}$ & $\mathrm{~N} / \mathrm{A}$ & $\mathrm{N} / \mathrm{A}$ & $\mathrm{N} / \mathrm{A}$ \\
\hline
\end{tabular}

TABLE 1. All the distributions with the ways to calculate their mean distance, mean squared distance and variance.

following equation:

$$
E\left(R_{n}^{2}\right)=n E\left(L^{2}\right)+2 E(L)^{2} \frac{c}{1-c}\left(n-\frac{1-c^{n}}{1-c}\right)
$$

or numerically (using individual based modelling with the chosen step length distribution) if either the variance or the mean do not exist and Eq. 2.1 is irrelevant. Here $E\left(L^{2}\right)$ is the mean square step length, $E(L)$ is the mean step length, $c$ is related to the possible turning angles of the particle and $n$ is the number of steps along the movement path; see [13] for details.

We will be looking at the following distributions. For the distributions based around $e^{-a x^{\mu}}$ we will focus on when $\mu=2$, the half-normal distribution, $\mu=1$, the exponential distribution, $\mu=1.5, \mu=0.5$ and $\mu=\frac{1}{3}$, the last three are a spread of distributions from the exponential family. The $(x+h)^{-\mu}$ based distributions have a general solution which is seen in table 1. As the distributions based on $\left(x^{\mu}+h^{\mu}\right)^{-1}$ are similar to the exponential family and cannot be generalized, we will be focusing our attention on four different values for $\mu: \mu=6, \mu=4, \mu=3$ and $\mu=2$, the last two are fat-tailed distributions and thus will need to be simulated to compare them to the other distributions.

The first two ways in which we will be comparing distributions will be 'standard', i.e. by changing the different parameters, $\sigma, \lambda, a$ or $h$, to give them equal square mean or variance. In order to include fattertailed distribution where the variance does not exist, we also consider a third way to compare distributions which will be based on equating the mean. Finally, aiming to further extend the list to include the fattailed distributions where the mean does not exist either, we consider a fourth way where we will be changing the parameters to equate the characteristic distance $y$ defined by the following equation:

$$
\int_{0}^{y} P(x, a) d x=C
$$

where parameter $C$ is therefore the probability to find the dispersing animal within the given distance $y$ from its original location (i.e. before dispersal), cf. $[32,33]$. In the context of a biological system - say a natural reserve or nature protection area - $C$ can be defined by an acceptable risk that the dispersing animal will leave the safe area of the size $y$. 


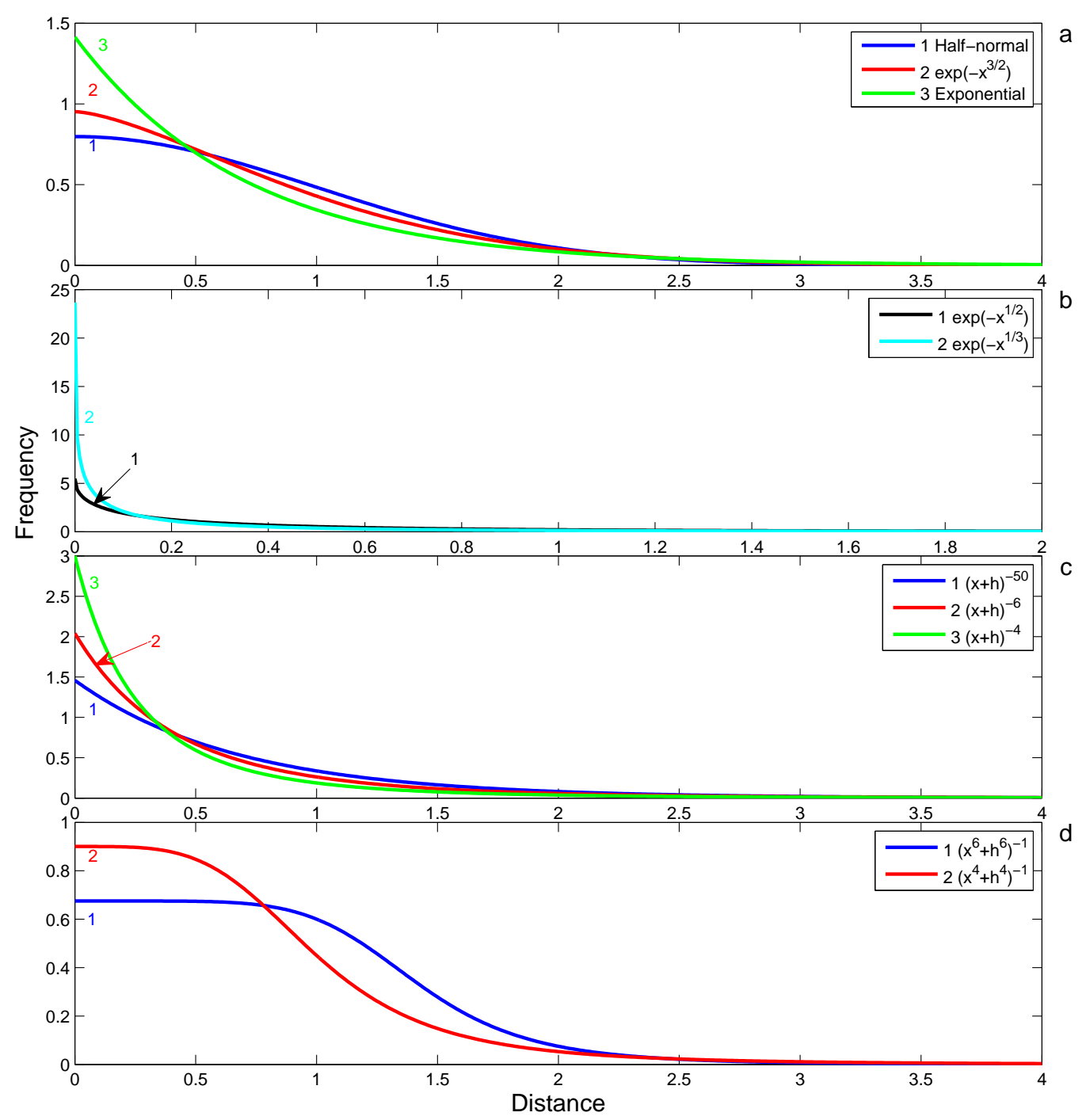

Figure 1. Plots of the frequency of all the distributions when they all have variance equal to one. Part a and $\mathrm{b}$ show the distributions based on $e^{-x^{\mu}}$. Part c shows the distributions based on $(x+h)^{-\mu}$. Part d shows the distributions based on $\left(x^{\mu}+h^{\mu}\right)^{-1}$.

Equation 2.2 gives the formula for working out the parameters of the compared step length distributions when using this method. $P(x, a)$ is the distribution being compared, $a$ is the parameter of the distribution, $x$ is the step length and $y$ is the limit to the step length. This calculates the frequency of the step lengths between 0 and $y$ and makes it equal to constant, $C$. Obviously for some distributions the square mean and variance do not exist, i.e. is $\infty$, for $\frac{\mu-1}{h^{1-\mu}}(x+h)^{-\mu}$ where $\mu \leq 3, \frac{3 \sqrt{3} h^{2}}{2 \pi}\left(x^{3}+h^{3}\right)^{-1}$ and $\frac{2 h}{\pi}\left(x^{2}+h^{2}\right)^{-1}$, and even the mean does not exist for $\frac{\mu-1}{h^{1-\mu}}(x+h)^{-\mu}$ where $\mu \leq 2$ and $\frac{2 h}{\pi}\left(x^{2}+h^{2}\right)^{-1}$. So the fourth method is already looking like a better way for comparing these distributions. 


\begin{tabular}{|c|c|c|c|c|c|}
\hline Distributions & Parameter & $\begin{array}{c}\text { Mean Distance } \\
-E(x) \\
\end{array}$ & $\begin{array}{c}\text { Mean Squared } \\
\text { Distance - } E\left(x^{2}\right) \\
\end{array}$ & Variance & Fastest \\
\hline $\begin{array}{l}\frac{\sqrt{2}}{\sigma \sqrt{\pi}} e^{-\frac{x^{2}}{2 \sigma^{2}}} \text { or } \\
\text { Half-Normal }\end{array}$ & $\sigma=\sqrt{\frac{\pi}{\pi-2}}$ & $\sqrt{\frac{2}{\pi-2}} \approx 1.324$ & $\frac{\pi}{\pi-2} \approx 2.752$ & 1 & 2nd \\
\hline$\frac{a^{\frac{2}{3}}}{\Gamma\left(\frac{5}{3}\right)} e^{-a x^{1.5}}$ & $a=\left(\frac{6 \Gamma\left(\frac{5}{3}\right)-4\left(\Gamma\left(\frac{4}{3}\right)\right)^{2}}{9\left(\Gamma\left(\frac{5}{3}\right)\right)^{2}}\right)^{\frac{3}{4}}$ & $\begin{array}{l}\frac{2 \Gamma\left(\frac{4}{3}\right)}{\sqrt{6 \Gamma\left(\frac{5}{3}\right)-4\left(\Gamma\left(\frac{4}{3}\right)\right)^{2}}} \\
\approx 1.197\end{array}$ & $\begin{array}{l}\frac{6 \Gamma\left(\frac{5}{3}\right)}{6 \Gamma\left(\frac{5}{3}\right)-4\left(\Gamma\left(\frac{4}{3}\right)\right)^{2}} \\
\quad \approx 2.432\end{array}$ & 1 & $3 \mathrm{rd}$ \\
\hline $\begin{array}{c}\lambda e^{-\lambda x} \text { or } \\
\text { Exponential }\end{array}$ & $\lambda=1$ & 1 & 2 & 1 & 4 th \\
\hline$\frac{a^{2}}{2} e^{-a \sqrt{x}}$ & $a=\sqrt[4]{84}$ & $\sqrt{\frac{3}{7}} \approx 0.6546$ & $\frac{10}{7} \approx 1.429$ & 1 & 6 th \\
\hline$\frac{a^{3}}{6} e^{-a \sqrt[3]{x}}$ & $a=\sqrt[6]{16560}$ & $\sqrt{\frac{5}{23}} \approx 0.4663$ & $\frac{28}{25}=1.12$ & 1 & 7 th \\
\hline $\begin{array}{l}\frac{\mu-1}{h^{1-\mu}}(x+h)^{-\mu} \text { or } \\
\text { Power-Law } \mu>3\end{array}$ & $\begin{array}{c}h=(\mu-2) \cdot \\
\sqrt{(\mu-3)(\mu-1)}\end{array}$ & $\sqrt{\frac{\mu-3}{\mu-1}}<1$ & $1<\frac{2 \mu-4}{\mu-1}<2$ & 1 & $\begin{array}{c}\text { Between } \\
4 \text { th and 8th }\end{array}$ \\
\hline $\begin{array}{l}\frac{\mu-1}{h^{1-\mu}}(x+h)^{-\mu} \text { or } \\
\text { Power-Law } \mu \leq 3\end{array}$ & $\mathrm{~N} / \mathrm{A}$ & $\mathrm{N} / \mathrm{A}$ & $\mathrm{N} / \mathrm{A}$ & $\mathrm{N} / \mathrm{A}$ & $\mathrm{N} / \mathrm{A}$ \\
\hline$\frac{3 h^{5}}{\pi}\left(x^{6}+h^{6}\right)^{-1}$ & $h=\sqrt{6}$ & $\sqrt{2} \approx 1.414$ & 3 & 1 & $1 \mathrm{st}$ \\
\hline$\frac{2^{\frac{3}{2}} h^{3}}{\pi}\left(x^{4}+h^{4}\right)^{-1}$ & $h=\sqrt{2}$ & 1 & 2 & 1 & 4 th \\
\hline$\frac{3 \sqrt{3} h^{2}}{2 \pi}\left(x^{3}+h^{3}\right)^{-1}$ & $\mathrm{~N} / \mathrm{A}$ & $\mathrm{N} / \mathrm{A}$ & $\mathrm{N} / \mathrm{A}$ & $\mathrm{N} / \mathrm{A}$ & $\mathrm{N} / \mathrm{A}$ \\
\hline$\frac{2 h}{\pi}\left(x^{2}+h^{2}\right)^{-1}$ & $\mathrm{~N} / \mathrm{A}$ & $\mathrm{N} / \mathrm{A}$ & $\mathrm{N} / \mathrm{A}$ & $\mathrm{N} / \mathrm{A}$ & $\mathrm{N} / \mathrm{A}$ \\
\hline
\end{tabular}

TABLE 2. The mean distance, mean squared distance and variance for various distributions based on $e^{-a x^{\mu}},(x+h)^{-\mu}$ and $\left(x^{\mu}+h^{\mu}\right)^{-1}$ with variance equal to one. The rightmost column gives the fastest distributions under the equal variance condition 


\begin{tabular}{|c|c|c|c|c|c|}
\hline Distributions & Parameter & $\begin{array}{l}\text { Mean Distance } \\
-E(x)\end{array}$ & $\begin{array}{c}\text { Mean Squared } \\
\text { Distance - } E\left(x^{2}\right)\end{array}$ & Variance & Fastest \\
\hline $\begin{array}{l}\frac{\sqrt{2}}{\sigma \sqrt{\pi}} e^{-\frac{x^{2}}{2 \sigma^{2}}} \text { or } \\
\text { Half-Normal }\end{array}$ & $\sigma=\sqrt{\frac{\pi}{2}}$ & 1 & $\frac{\pi}{2} \approx 1.571$ & $\frac{\pi}{2}-1^{2} \approx 0.5708$ & 7 th \\
\hline$\frac{a^{\frac{2}{3}}}{\Gamma\left(\frac{5}{3}\right)} e^{-a x^{1.5}}$ & $a=\left(\frac{2 \Gamma\left(\frac{4}{3}\right)}{3 \Gamma\left(\frac{5}{3}\right)}\right)^{\frac{3}{2}}$ & 1 & $\frac{3 \Gamma\left(\frac{5}{3}\right)}{2\left(\Gamma\left(\frac{4}{3}\right)\right)^{2}} \approx 1.698$ & $\begin{array}{l}\frac{3 \Gamma\left(\frac{5}{3}\right)}{2\left(\Gamma\left(\frac{4}{3}\right)\right)^{2}}-1^{2} \\
\quad \approx 0.6981\end{array}$ & 6 th \\
\hline $\begin{array}{c}\lambda e^{-\lambda x} \text { or } \\
\text { Exponential }\end{array}$ & $\lambda=1$ & 1 & 2 & 1 & 4 th \\
\hline$\frac{a^{2}}{2} e^{-a \sqrt{x}}$ & $a=\sqrt{6}$ & 1 & $\frac{10}{3} \approx 3.333$ & $\frac{7}{3} \approx 2.333$ & $3 \mathrm{rd}$ \\
\hline$\frac{a^{3}}{6} e^{-a \sqrt[3]{x}}$ & $a=\sqrt[3]{60}$ & 1 & $\frac{28}{5}=5.6$ & $\frac{23}{5}=4.6$ & 2nd \\
\hline $\begin{array}{l}\frac{\mu-1}{h^{1-\mu}}(x+h)^{-\mu} \text { or } \\
\text { Power-Law } \mu \geq 3\end{array}$ & $1 \leq h=\mu-2$ & 1 & $2<\frac{2 \mu-4}{\mu-3}$ & $1<\frac{\mu-1}{\mu-3}$ & $\begin{array}{c}\text { Between } \\
\text { 1st and 4th }\end{array}$ \\
\hline $\begin{array}{l}\frac{\mu-1}{h^{1-\mu}}(x+h)^{-\mu} \text { or } \\
\text { Power-Law } \mu<3\end{array}$ & $\mathrm{~N} / \mathrm{A}$ & $\mathrm{N} / \mathrm{A}$ & $\mathrm{N} / \mathrm{A}$ & $\mathrm{N} / \mathrm{A}$ & $\mathrm{N} / \mathrm{A}$ \\
\hline$\frac{3 h^{5}}{\pi}\left(x^{6}+h^{6}\right)^{-1}$ & $h=\sqrt{3}$ & 1 & $\frac{3}{2}=1.5$ & 0.5 & 8 th \\
\hline$\frac{2^{\frac{3}{2}} h^{3}}{\pi}\left(x^{4}+h^{4}\right)^{-1}$ & $h=\sqrt{2}$ & 1 & 2 & 1 & 4 th \\
\hline$\frac{3 \sqrt{3} h^{2}}{2 \pi}\left(x^{3}+h^{3}\right)^{-1}$ & $h=1$ & 1 & $\infty$ & $\infty$ & 1 st \\
\hline$\frac{2 h}{\pi}\left(x^{2}+h^{2}\right)^{-1}$ & $\mathrm{~N} / \mathrm{A}$ & $\mathrm{N} / \mathrm{A}$ & $\mathrm{N} / \mathrm{A}$ & $\mathrm{N} / \mathrm{A}$ & $\mathrm{N} / \mathrm{A}$ \\
\hline
\end{tabular}

TABLE 3. The mean distance, mean squared distance and variance for distributions based on $e^{-a x^{1.5}}, e^{-a \sqrt{x}}, e^{-a \sqrt[3]{x}}$, $\left(x^{4}+h^{4}\right)^{-1},\left(x^{6}+h^{6}\right)^{-1},\left(x^{3}+h^{3}\right)^{-1}$ and $\left(x^{2}+h^{2}\right)^{-1}$ and the half-normal, exponential and standard power-law distributions and with mean distance equal to one. The rightmost column gives the fastest distributions under the equal mean condition. 


\begin{tabular}{|c|c|c|c|c|c|}
\hline Distributions & Parameter & $\begin{array}{c}\text { Mean Distance } \\
-E(x)\end{array}$ & $\begin{array}{c}\text { Mean Squared } \\
\text { Distance - } E\left(x^{2}\right)\end{array}$ & Variance & Fastest \\
\hline $\begin{array}{l}\frac{\sqrt{2}}{\sigma \sqrt{\pi}} e^{-\frac{x^{2}}{2 \sigma^{2}}} \text { or } \\
\text { Half-Normal }\end{array}$ & $\sigma=1$ & $\sqrt{\frac{2}{\pi}} \approx 0.7979$ & 1 & $1-\frac{2}{\pi} \approx 0.3634$ & 2 nd \\
\hline$\frac{a^{\frac{2}{3}}}{\Gamma\left(\frac{5}{3}\right)} e^{-a x^{1.5}}$ & $a=\left(\frac{2}{3 \Gamma\left(\frac{5}{3}\right)}\right)^{\frac{3}{4}}$ & $\begin{array}{l}\frac{2 \Gamma\left(\frac{4}{3}\right)}{\sqrt{6 \Gamma\left(\frac{5}{3}\right)}} \\
\approx 0.7674\end{array}$ & 1 & $\begin{array}{c}1-\left(\frac{2 \Gamma\left(\frac{4}{3}\right)}{\sqrt{6 \Gamma\left(\frac{5}{3}\right)}}\right)^{2} \\
\approx 0.4111\end{array}$ & $3 \mathrm{rd}$ \\
\hline $\begin{array}{c}\lambda e^{-\lambda x} \text { or } \\
\text { Exponential }\end{array}$ & $\lambda=\sqrt{2}$ & $\frac{1}{\sqrt{2}} \approx 0.7071$ & 1 & $1-\frac{1}{2}=0.5$ & 4 th \\
\hline$\frac{a^{2}}{2} e^{-a \sqrt{x}}$ & $a=\sqrt[4]{120}$ & $\sqrt{\frac{3}{10}} \approx 0.5477$ & 1 & $1-\frac{3}{10}=0.7$ & 6 th \\
\hline$\frac{a^{3}}{6} e^{-a \sqrt[3]{x}}$ & $a=\sqrt[6]{20160}$ & $\sqrt{\frac{5}{28}} \approx 0.4226$ & 1 & $1-\frac{5}{28} \approx 0.8214$ & 7 th \\
\hline $\begin{array}{l}\frac{\mu-1}{h^{1-\mu}}(x+h)^{-\mu} \text { or } \\
\text { Power-Law } \mu>3\end{array}$ & $\begin{array}{c}0<h \\
=\sqrt{\frac{(\mu-2)(\mu-3)}{2}}\end{array}$ & $\begin{aligned} & \sqrt{\frac{\mu-3}{2(\mu-2)}} \\
< & \frac{1}{\sqrt{2}} \approx 0.7071\end{aligned}$ & 1 & $\begin{array}{c}\frac{1}{2}<1-\frac{\mu-3}{2(\mu-2)} \\
=\frac{\mu-1}{2(\mu-2)}<1\end{array}$ & $\begin{array}{c}\text { Between } \\
4 \text { th and } 7 \text { th }\end{array}$ \\
\hline $\begin{array}{l}\frac{\mu-1}{h^{1-\mu}}(x+h)^{-\mu} \text { or } \\
\text { Power-Law } \mu \leq 3\end{array}$ & $\mathrm{~N} / \mathrm{A}$ & $\mathrm{N} / \mathrm{A}$ & $\mathrm{N} / \mathrm{A}$ & $\mathrm{N} / \mathrm{A}$ & $\mathrm{N} / \mathrm{A}$ \\
\hline$\frac{3 h^{5}}{\pi}\left(x^{6}+h^{6}\right)^{-1}$ & $h=\sqrt{2}$ & $\sqrt{\frac{2}{3}} \approx 0.8165$ & 1 & $1-\frac{2}{3}=0 . \dot{3}$ & $1 \mathrm{st}$ \\
\hline$\frac{2^{\frac{3}{2}} h^{3}}{\pi}\left(x^{4}+h^{4}\right)^{-1}$ & $h=1$ & $\frac{1}{\sqrt{2}} \approx 0.7071$ & 1 & $1-\frac{1}{2}=0.5$ & 4 th \\
\hline$\frac{3 \sqrt{3} h^{2}}{2 \pi}\left(x^{3}+h^{3}\right)^{-1}$ & $\mathrm{~N} / \mathrm{A}$ & $\mathrm{N} / \mathrm{A}$ & $\mathrm{N} / \mathrm{A}$ & $\mathrm{N} / \mathrm{A}$ & $\mathrm{N} / \mathrm{A}$ \\
\hline$\frac{2 h}{\pi}\left(x^{2}+h^{2}\right)^{-1}$ & $\mathrm{~N} / \mathrm{A}$ & $\mathrm{N} / \mathrm{A}$ & $\mathrm{N} / \mathrm{A}$ & $\mathrm{N} / \mathrm{A}$ & $\mathrm{N} / \mathrm{A}$ \\
\hline
\end{tabular}

TABLE 4. The mean distance, mean squared distance and variance for distributions based on $e^{-a x^{1.5}}, e^{-a \sqrt{x}}, e^{-a \sqrt[3]{x}}$, $\left(x^{4}+h^{4}\right)^{-1},\left(x^{6}+h^{6}\right)^{-1},\left(x^{3}+h^{3}\right)^{-1}$ and $\left(x^{2}+h^{2}\right)^{-1}$ and the half-normal, exponential and standard power-law distributions and with mean squared distance equal to 1 . The rightmost column gives the fastest distributions under the mean distance condition. So distance will only be different under persistent movement and all distributions will be the same if all angles around the circle have equal chance of being chosen. 


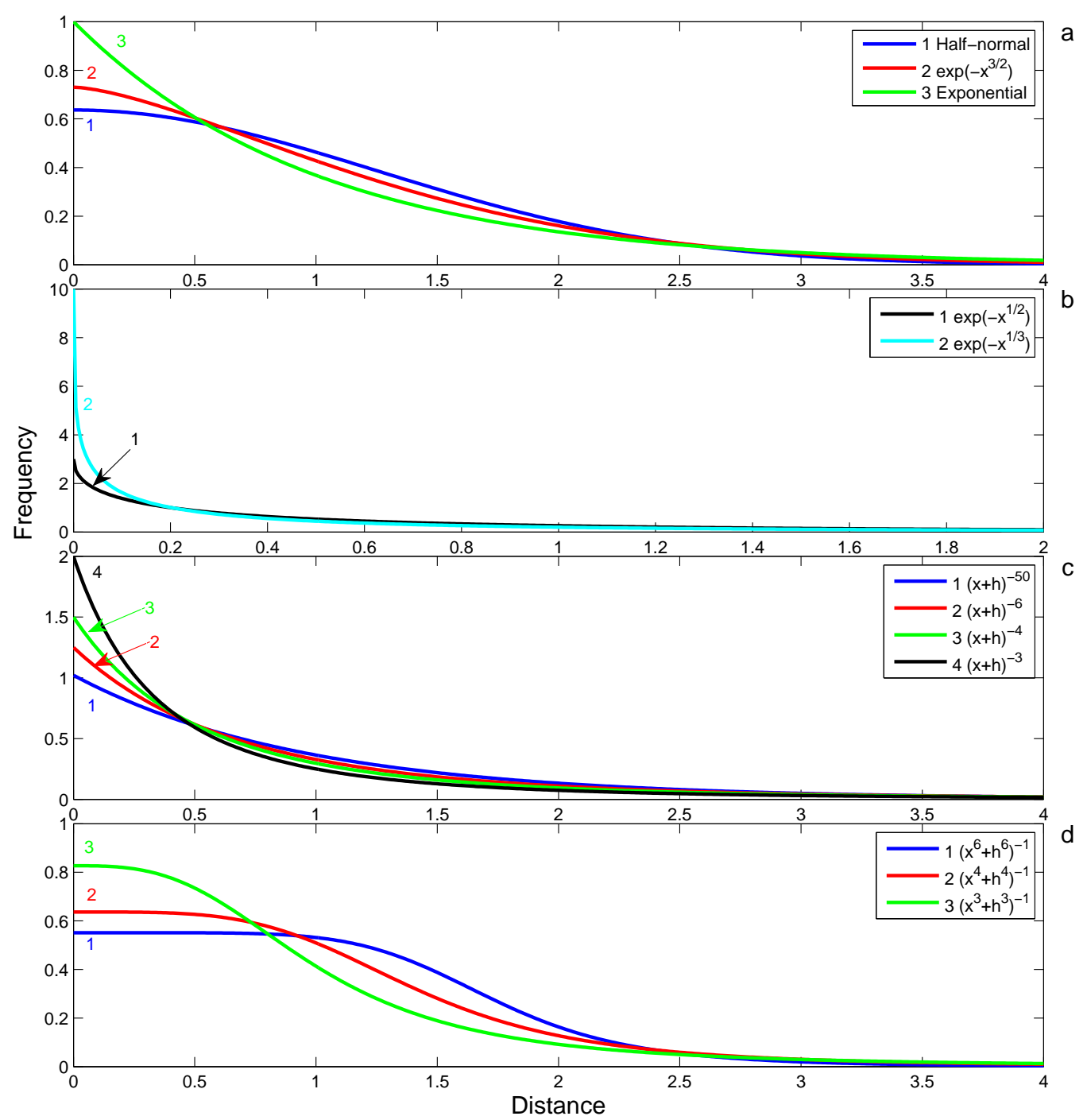

Figure 2. Plots of the frequency of all the distributions when they all have mean equal to one. Part a and $\mathrm{b}$ show the distributions based on $e^{-x^{\mu}}$. Part $\mathrm{c}$ shows the distributions based on $(x+h)^{-\mu}$. Part d shows the distributions based on $\left(x^{\mu}+h^{\mu}\right)^{-1}$.

\section{Results}

Tables 2, 3 and 4 show the mean, square mean and variance for when the variance, mean distance and mean square distance are equal respectively. Figures 1, 2 and 3 show the step length distributions in the cases where they were equated using the same variance (Fig.1), the same mean (Fig.2) and the same square mean (Fig.3); see tables 2, 3 and 4 respectively.

Table 5 shows the parameter, mean and square mean when the integral is equal to one half and Table 6 shows the parameter and variance when the integral is equal to one half. This parameter is then used to find the mean distance, mean squared distance and variance so that the distributions can be compared easily. Figures 4, 5 and 6 show how the distributions look when the parameters are set to different values of $C$, which are shown in the tables $5, .2$ and .3 respectively. 


\begin{tabular}{|c|c|c|c|}
\hline Distributions & $y=1$ and $C=\frac{1}{2}$ & $\begin{array}{l}\text { Mean Distance } \\
-E(x) \\
\end{array}$ & $\begin{array}{l}\text { Mean Squared Distance } \\
-E\left(x^{2}\right) \\
\end{array}$ \\
\hline $\begin{array}{l}\frac{\sqrt{2}}{\sigma \sqrt{\pi}} e^{-\frac{x^{2}}{2 \sigma^{2}}} \text { or } \\
\text { Half-Normal }\end{array}$ & $\sigma=\left(\sqrt{2} e r f^{-1}\left(\frac{1}{2}\right)\right)^{-1}$ & $\frac{1}{\sqrt{\pi} \operatorname{erf} f^{-1}\left(\frac{1}{2}\right)} \approx 1.183$ & $\frac{1}{2\left(e r f^{-1}\left(\frac{1}{2}\right)\right)^{2}} \approx 2.198$ \\
\hline$\frac{a^{\frac{2}{3}}}{\Gamma\left(\frac{5}{3}\right)} e^{-a x^{1.5}}$ & $a=\left(\Gamma^{-1}\left(\frac{2}{3}, \frac{1}{2}\left(\Gamma\left(\frac{2}{3}\right)\right)\right)\right)$ & $\begin{array}{c}\frac{2 \Gamma\left(\frac{4}{3}\right)}{3\left(\Gamma^{-1}\left(\frac{2}{3}, \frac{1}{2}\left(\Gamma\left(\frac{2}{3}\right)\right)\right)\right)^{\frac{2}{3}} \Gamma\left(\frac{5}{3}\right)} \\
\approx 1.266\end{array}$ & $\begin{array}{c}\frac{2}{3\left(\Gamma^{-1}\left(\frac{2}{3}, \frac{1}{2}\left(\Gamma\left(\frac{2}{3}\right)\right)\right)\right)^{\frac{4}{3}} \Gamma\left(\frac{5}{3}\right)} \\
\approx 2.716\end{array}$ \\
\hline $\begin{array}{c}\lambda e^{-\lambda x} \text { or } \\
\text { Exponential }\end{array}$ & $\lambda=\ln (2)$ & $\frac{1}{\ln (2)} \approx 1.443$ & $\frac{2}{\ln (4)} \approx 4.163$ \\
\hline$\frac{a^{2}}{2} e^{-a \sqrt{x}}$ & $a=\left(W\left(\frac{-1}{2 e}\right)+1\right)$ & $\frac{6}{\left(W\left(\frac{-1}{2 e}\right)+1\right)^{2}} \approx 2.130$ & $\frac{120}{\left(W\left(\frac{-1}{2 e}\right)+1\right)^{4}} \approx 15.12$ \\
\hline$\frac{a^{3}}{6} e^{-a \sqrt[3]{x}}$ & $a \approx 2.674$ & $\approx 3.138$ & $\approx 55.15$ \\
\hline $\begin{array}{l}\frac{\mu-1}{h^{1-\mu}}(x+h)^{-\mu} \text { or } \\
\text { Power-Law } \mu>3\end{array}$ & $\begin{array}{l}(\sqrt{2}-1)^{-1}<h \\
\left(\left(\frac{1}{2}\right)^{\frac{1}{1-\mu}}-1\right)^{-1}\end{array}$ & $\frac{1}{(\mu-2)\left(\left(\frac{1}{2}\right)^{\frac{1}{1-\mu}}-1\right)}<2.414$ & $\frac{2}{(\mu-3)(\mu-2)\left(\left(\frac{1}{2}\right)^{\frac{1}{1-\mu}}-1\right)^{2}}$ \\
\hline $\begin{array}{l}2 h^{2}(x+h)^{-3} \text { or } \\
\text { Power-Law } \mu=3\end{array}$ & $h=(\sqrt{2}-1)^{-1}$ & $\frac{1}{(\sqrt{2}-1)} \approx 2.414$ & $\mathrm{~N} / \mathrm{A}$ \\
\hline $\begin{array}{c}h(x+h)^{-2} \text { or } \\
\text { Power-Law } \mu=2\end{array}$ & $h=1$ & $\mathrm{~N} / \mathrm{A}$ & $\mathrm{N} / \mathrm{A}$ \\
\hline$\frac{3 h^{5}}{\pi}\left(x^{6}+h^{6}\right)^{-1}$ & $h \approx 1.904$ & $\approx 1.099$ & $\approx 1.813$ \\
\hline$\frac{2^{\frac{3}{2}} h^{3}}{\pi}\left(x^{4}+h^{4}\right)^{-1}$ & $h \approx 1.766$ & $\approx 1.248$ & $\approx 3.117$ \\
\hline$\frac{3 \sqrt{3} h^{2}}{2 \pi}\left(x^{3}+h^{3}\right)^{-1}$ & $h \approx 1.559$ & $\approx 1.559$ & $\mathrm{~N} / \mathrm{A}$ \\
\hline$\frac{2 h}{\pi}\left(x^{2}+h^{2}\right)^{-1}$ & $h=1$ & $\mathrm{~N} / \mathrm{A}$ & $\mathrm{N} / \mathrm{A}$ \\
\hline
\end{tabular}

TABLE 5. The case when the integration between 0 and y is equal to a constant, $C=\frac{1}{2}$. We have the values of the parameter, mean distance and square mean distance for distributions based on $e^{-a x^{1.5}}, e^{-a \sqrt{x}}, e^{-a \sqrt[3]{x}},\left(x^{4}+h^{4}\right)^{-1},\left(x^{6}+h^{6}\right)^{-1}$, $\left(x^{3}+h^{3}\right)^{-1}$ and $\left(x^{2}+h^{2}\right)^{-1}$ and the half-normal, exponential and standard power-law distributions. 


\begin{tabular}{|c|c|c|c|}
\hline Distributions & $y=1$ and $C=\frac{1}{2}$ & Variance & Fastest \\
\hline $\begin{array}{l}\frac{\sqrt{2}}{\sigma \sqrt{\pi}} e^{-\frac{x^{2}}{2 \sigma^{2}}} \text { or } \\
\text { Half-Normal }\end{array}$ & $\sigma=\left(\sqrt{2} e r f^{-1}\left(\frac{1}{2}\right)\right)^{-1}$ & $\frac{\pi-2}{2 \pi\left(e r f^{-1}\left(\frac{1}{2}\right)\right)^{2}} \approx 0.7987$ & 11 th \\
\hline$\frac{a^{\frac{2}{3}}}{\Gamma\left(\frac{5}{3}\right)} e^{-a x^{1.5}}$ & $a=\left(\Gamma^{-1}\left(\frac{2}{3}, \frac{1}{2}\left(\Gamma\left(\frac{2}{3}\right)\right)\right)\right)$ & $\begin{array}{c}\frac{6 \Gamma\left(\frac{5}{3}\right)-2 \Gamma\left(\frac{4}{3}\right)}{9\left(\Gamma^{-1}\left(\frac{2}{3}, \frac{1}{2}\left(\Gamma\left(\frac{2}{3}\right)\right)\right)\right)^{\frac{4}{3}}\left(\Gamma\left(\frac{5}{3}\right)\right)^{2}} \\
\approx 1.117\end{array}$ & 10 th \\
\hline $\begin{array}{c}\lambda e^{-\lambda x} \text { or } \\
\text { Exponential }\end{array}$ & $\lambda=\ln (2)$ & $\frac{1}{\ln (4)} \approx 2.081$ & 8 th \\
\hline$\frac{a^{2}}{2} e^{-a \sqrt{x}}$ & $a=\left(W\left(\frac{-1}{2 e}\right)+1\right)$ & $\frac{84}{\left(W\left(\frac{-1}{2 e}\right)+1\right)^{4}} \approx 10.59$ & 6 th \\
\hline$\frac{a^{3}}{6} e^{-a \sqrt[3]{x}}$ & $a \approx 2.674$ & $\approx 45.30$ & 5 th \\
\hline $\begin{array}{l}\frac{\mu-1}{h^{1-\mu}}(x+h)^{-\mu} \text { or } \\
\text { Power-Law } \mu>3\end{array}$ & $\begin{array}{l}(\sqrt{2}-1)^{-1}<h \\
\left(\left(\frac{1}{2}\right)^{\frac{1}{1-\mu}}-1\right)^{-1}\end{array}$ & $\frac{\mu-1}{(\mu-3)(\mu-2)^{2}\left(\left(\frac{1}{2}\right)^{\frac{1}{1-\mu}}-1\right)^{2}}$ & $\begin{array}{c}\text { Between } \\
4 \text { th and } 13 \text { th }\end{array}$ \\
\hline $\begin{array}{c}2 h^{2}(x+h)^{-3} \text { or } \\
\text { Power-Law } \mu=3\end{array}$ & $h=(\sqrt{2}-1)^{-1}$ & $\mathrm{~N} / \mathrm{A}$ & $3 \mathrm{rd}$ \\
\hline $\begin{array}{c}h(x+h)^{-2} \text { or } \\
\text { Power-Law } \mu=2\end{array}$ & $h=1$ & $\mathrm{~N} / \mathrm{A}$ & $1 \mathrm{st}$ \\
\hline$\frac{3 h^{5}}{\pi}\left(x^{6}+h^{6}\right)^{-1}$ & $h \approx 1.904$ & $\approx 0.6044$ & 12 th \\
\hline$\frac{2^{\frac{3}{2}} h^{3}}{\pi}\left(x^{4}+h^{4}\right)^{-1}$ & $h \approx 1.766$ & $\approx 1.559$ & 9 th \\
\hline$\frac{3 \sqrt{3} h^{2}}{2 \pi}\left(x^{3}+h^{3}\right)^{-1}$ & $h \approx 1.559$ & $\mathrm{~N} / \mathrm{A}$ & 7 th \\
\hline$\frac{2 h}{\pi}\left(x^{2}+h^{2}\right)^{-1}$ & $h=1$ & $\mathrm{~N} / \mathrm{A}$ & 2nd \\
\hline
\end{tabular}

TABLE 6 . The case when the integration between 0 and y is equal to a constant, $C=\frac{1}{2}$. We have the values of the parameter and variance for distributions based on $e^{-a x^{1.5}}, e^{-a \sqrt{x}}, e^{-a \sqrt[3]{x}},\left(x^{4}+h^{4}\right)^{-1},\left(x^{6}+h^{6}\right)^{-1},\left(x^{3}+h^{3}\right)^{-1}$ and $\left(x^{2}+h^{2}\right)^{-1}$ and the half-normal, exponential and standard power-law distributions. 


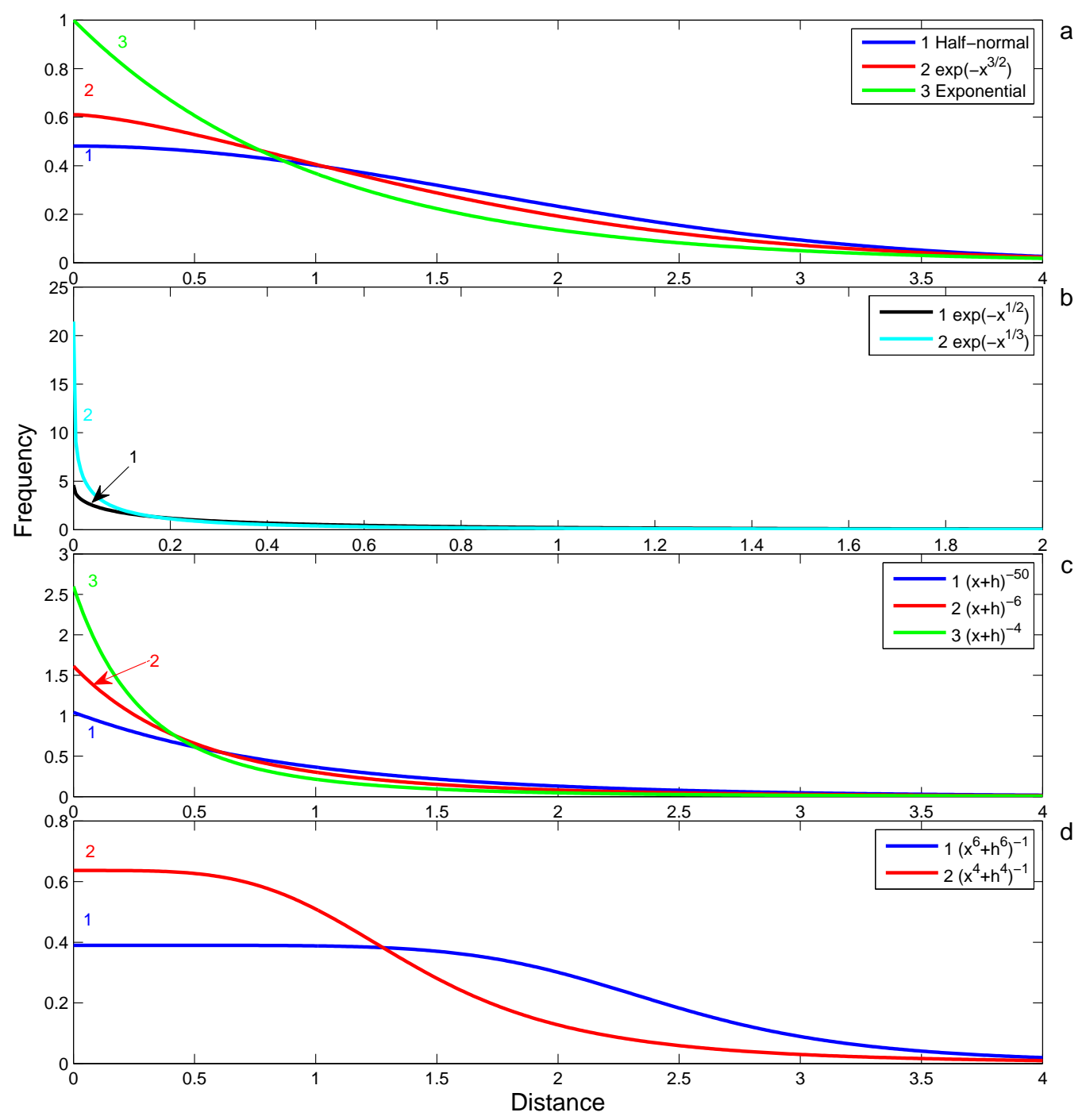

Figure 3. Plots of the frequency of all the distributions when they all have square mean equal to one. Part $\mathrm{a}$ and $\mathrm{b}$ show the distributions based on $e^{-x^{\mu}}$. Part $\mathrm{c}$ shows the distributions based on $(x+h)^{-\mu}$. Part d shows the distributions based on $\left(x^{\mu}+h^{\mu}\right)^{-1}$.

Figure 7 shows the displacement of various distributions from the exponential family on a log scale for time and displacement. It has no consistences in the direction and so the lines are straight which is to be expected, due to the mean squared displacement being equal to a constant multiplied by step number as $c=0$ for equation 2.1, [13]. When the direction of movement has consistency, i.e. figure 8 , the lines are more interesting and no longer straight.

Figure 9 and 10 shows the displacement of various distributions, based on $(x+h)^{-\mu}$ and $\left(x^{\mu}+h^{\mu}\right)^{-1}$, on a $\log$ scale for time and displacement. It has no consistences in the direction and so the lines are straight which is to be expected as before, for figure 7 . The erratic nature of the lines for the distribution based on $(x+h)^{-2}$ and $\left(x^{2}+h^{2}\right)^{-1}$ is due to these distributions having very fat tails, which means very 


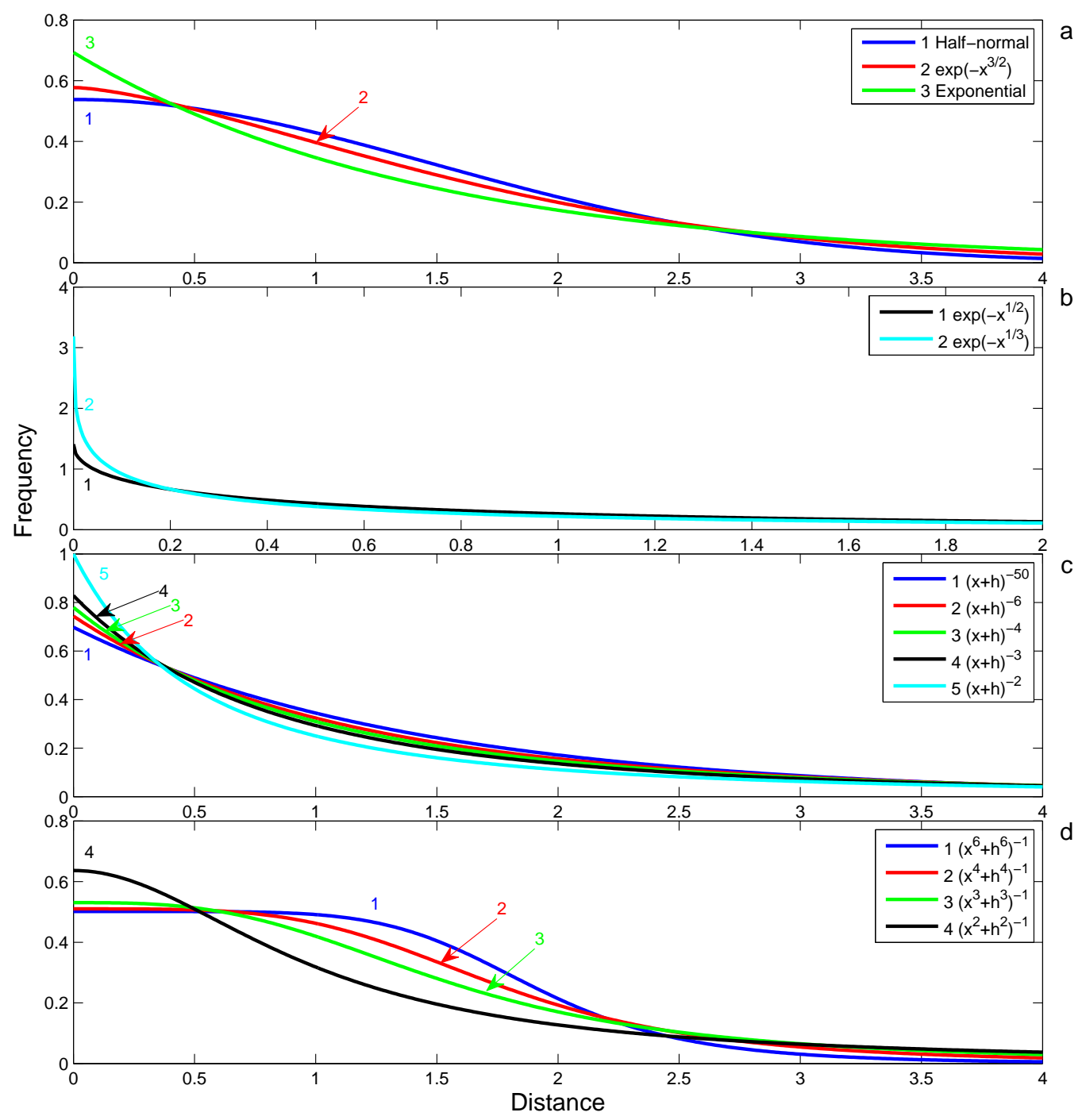

Figure 4. Plots of the frequency of all the distributions when they all have $y=1$ and $C=\frac{1}{2}$. Part a and b show the distributions based on $e^{-x^{\mu}}$. Part c shows the distributions based on $(x+h)^{-\mu}$. Part d shows the distributions based on $\left(x^{\mu}+h^{\mu}\right)^{-1}$.

high step lengths are relatively common. Thus vast numbers of simulations have to be run to smooth out the line.

\section{Discussion and conclusions}

In this paper, we considered several step length distributions (dispersal kernels) that are often used to describe individual movement of a 'particle' (such as animal, seed or pollen). We used several somewhat different ways to make the kernels equivalent by equating a chosen characteristic quantity or characteristic length. For each of those ways, we then calculated the MSD as a function of time and identified the dispersal kernel that corresponds to the fastest growing MSD. Interestingly and rather counter-intuitively, 


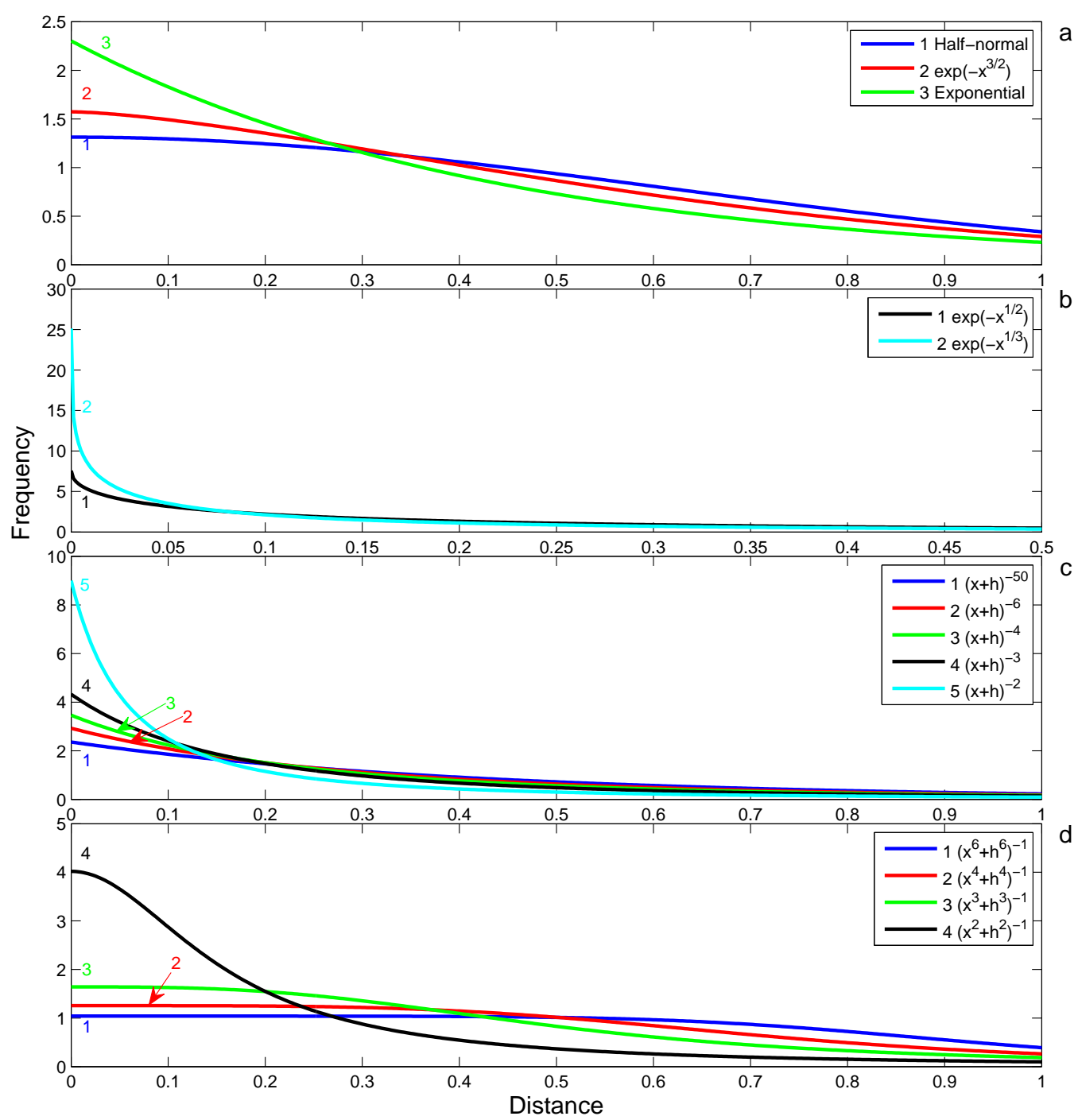

Figure 5. Plots of the frequency of all the distributions when they all have $y=1$ and $C=\frac{9}{10}$. Part a and $\mathrm{b}$ show the distributions based on $e^{-x^{\mu}}$. Part $\mathrm{c}$ shows the distributions based on $(x+h)^{-\mu}$. Part d shows the distributions based on $\left(x^{\mu}+h^{\mu}\right)^{-1}$.

we found that the kernel corresponding to the fastest growing MSD, and hence to the fastest dispersal, depends on how the kernels are equated. Hence the conclusion as to what is the 'most efficient' movement pattern appears to be relative rather than absolute. It can depend on the ecological context and/or the details of a specific habitat and species traits.

In particular, we mention here that, although the half-normal distribution is usually considered to be slower than other distributions, such as the exponential or standard power-laws, because it has a much faster rate of decay at large distances, if we equate the variance, table 2 , of such distributions then the half-normal is faster than other common distributions. This is interesting to note, as it is contrary to initial thoughts on distributions. However, if any other method of comparison, shown here, is used then the normal distribution is not the fastest. 


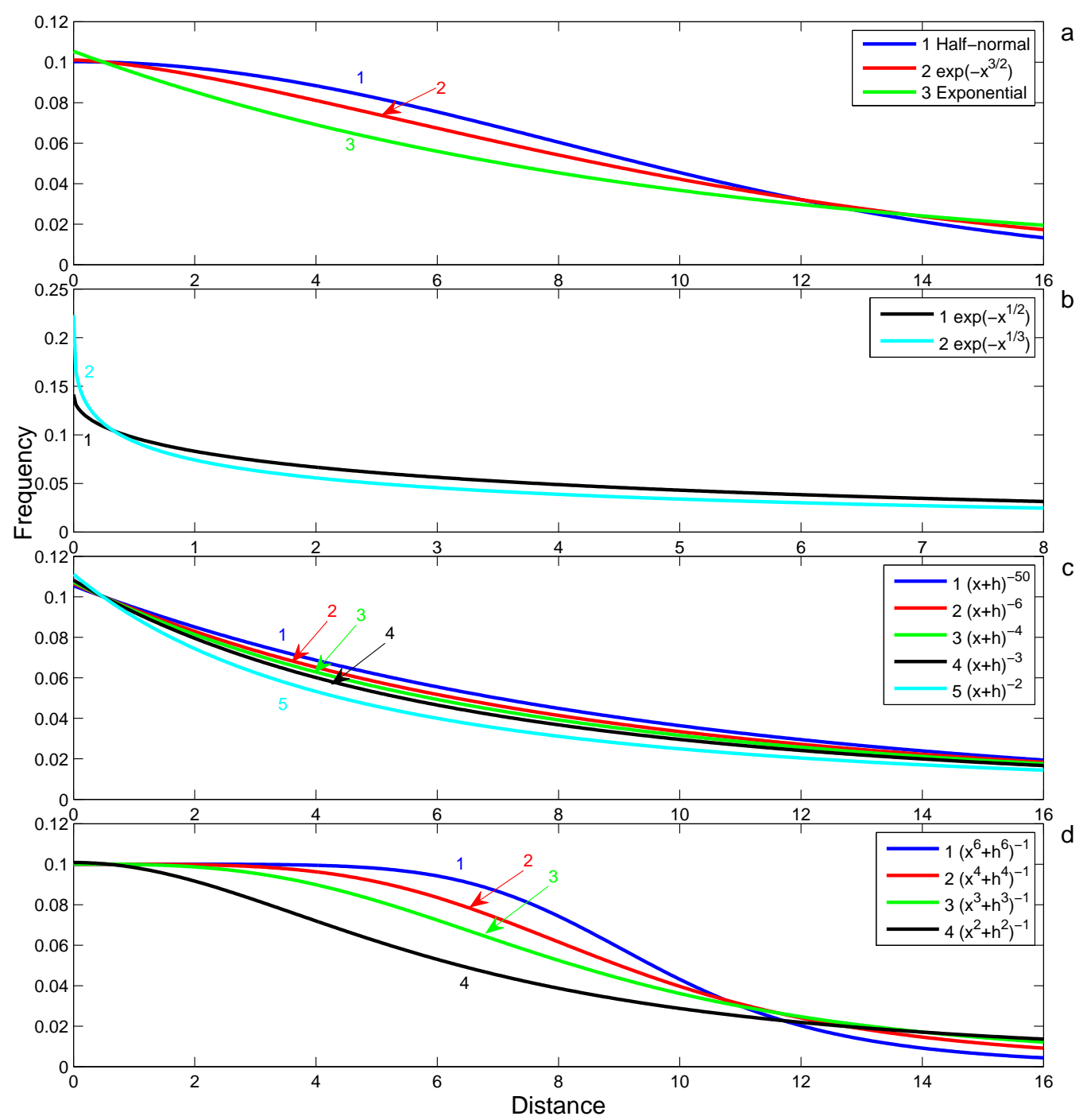

Figure 6. Plots of the frequency of all the distributions when they all have $y=1$ and $C=\frac{1}{10}$. Part a and $\mathrm{b}$ show the distributions based on $e^{-x^{\mu}}$. Part $\mathrm{c}$ shows the distributions based on $(x+h)^{-\mu}$. Part d shows the distributions based on $\left(x^{\mu}+h^{\mu}\right)^{-1}$.

The new method detailed in this paper for comparing distributions, (see Eq. 2.2 and the comments after the equation), is more applicable than other methods such as equating the mean or variance, as it can be applied to more distributions. However, the distributions this allows to be compared are fattailed and often questioned, [37-39], as to their relevance to step lengths. So this increase in the range of distributions able to be compared does not seem to be important. Despite this, there are other advantages to this method; such as no longer needing to calculate the expected step length which skews the earlier methods of comparison.

An interesting observation, if we look back at tables 2, 3 and 4 , is that we see the exponential distribution, $\lambda e^{-\lambda x}$, and the $\frac{2^{\frac{3}{2}} h^{3}}{\pi}\left(x^{4}+h^{4}\right)^{-1}$ distribution have the same mean, square mean and variance when compared in these ways. The power-law distribution, $\frac{\mu-1}{h^{1-\mu}}(x+h)^{-\mu}$, also has the same mean, square 


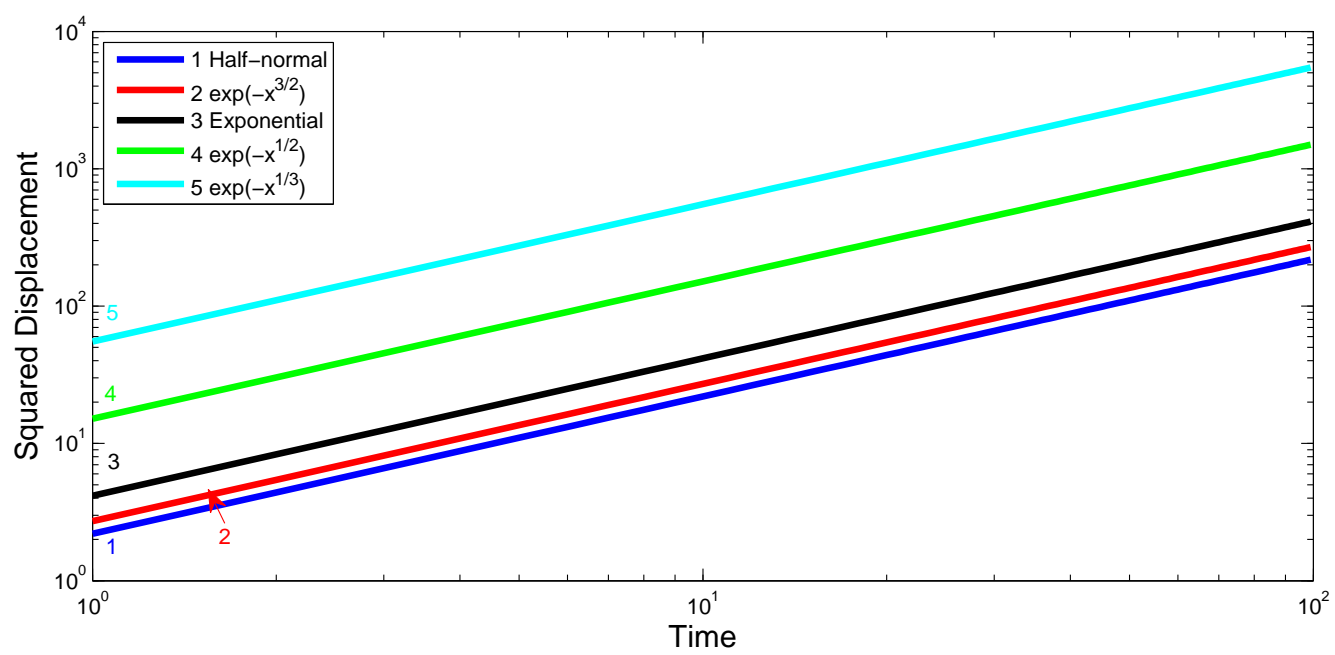

Figure 7. The predicted mean squared displacement, [13], for various distributions based on $e^{-a x^{\mu}}$ all with $y=1$ and $C=\frac{1}{2}$. There is no consistency to the direction of movement

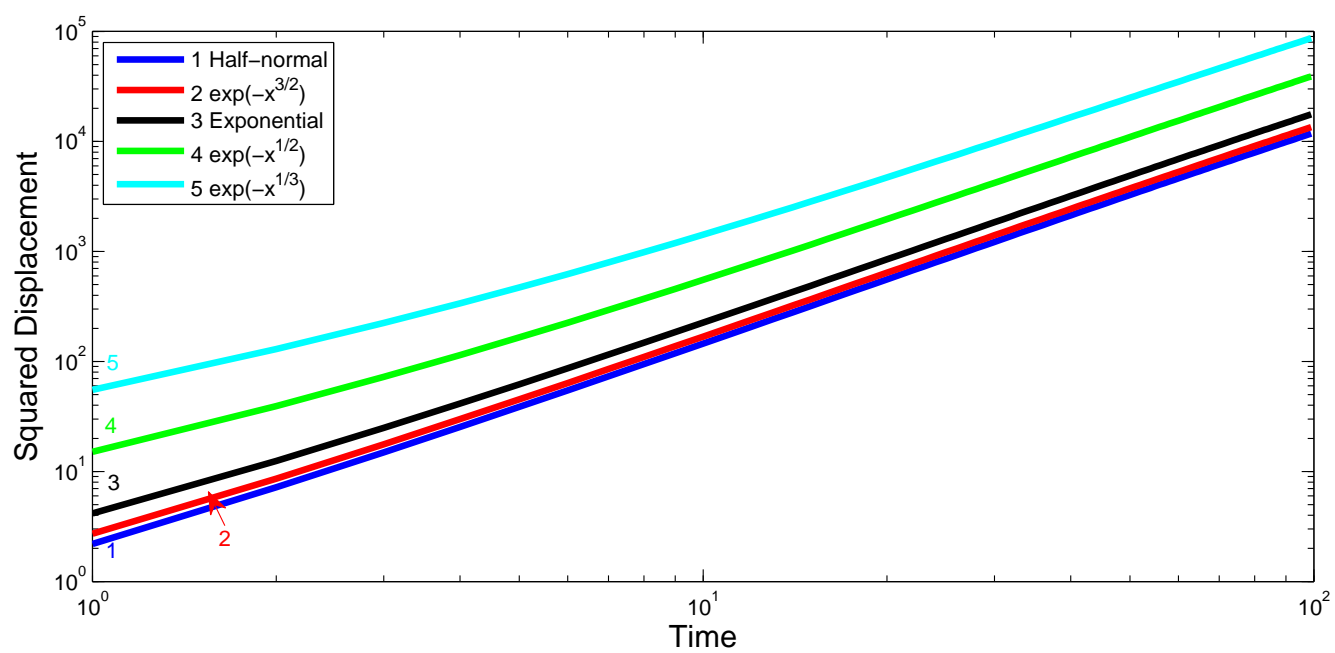

FiguRE 8. The predicted mean squared displacement, [13], for various distributions based on $e^{-a x^{\mu}}$ all with $y=1$ and $C=\frac{1}{2}$. Here there is quite high consistency to the direction of movement

mean and variance when compared in these ways when $\mu$ tends to infinity. Figures 1,2 and 3 show that the frequency of step lengths is very different, so while these distributions are very similar when choosing a distribution to base the step length on, they are crucially different and picking the 'wrong' one would impact the information that can be gained from such simulations. However, they are different when the integral between 0 and $y$ is equal, as seen in tables $5, .2$ and .3 .

Another thing of interest is that the order, fastest to slowest, of all the distributions is reversed between when they have equal variance and when they have equal mean. Also the order from when they have equal mean is kept for the square mean and mostly for when the integral between 0 and $y$ is equal. 


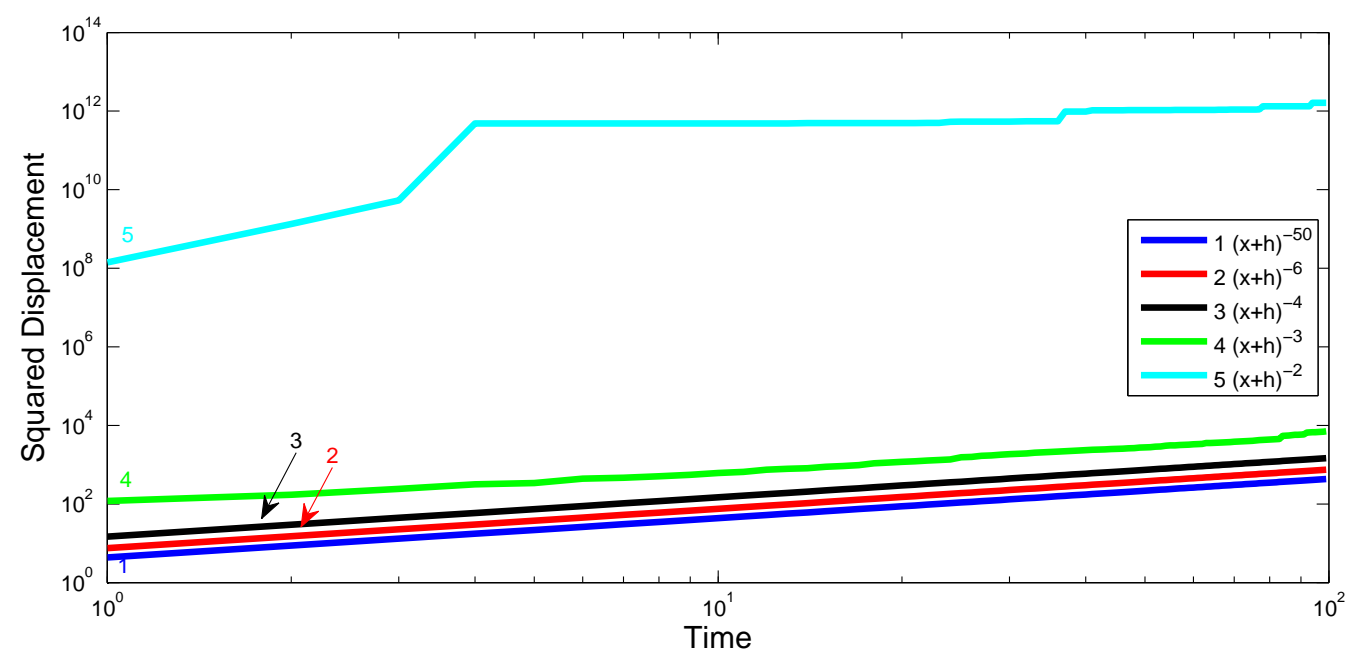

Figure 9. The predicted and estimated mean squared displacement, [13], for various distributions based on $(x+h)^{-\mu}$ all with $y=1$ and $C=\frac{1}{2}$. There is no consistency to the direction of movement. The lines for the lower powers, i.e. $(x+h)^{-3}$ and $(x+h)^{-2}$, are estimated by averaging a large number of randomly generated paths, the other lines are produced using the MSD described by Eq. (2.1).

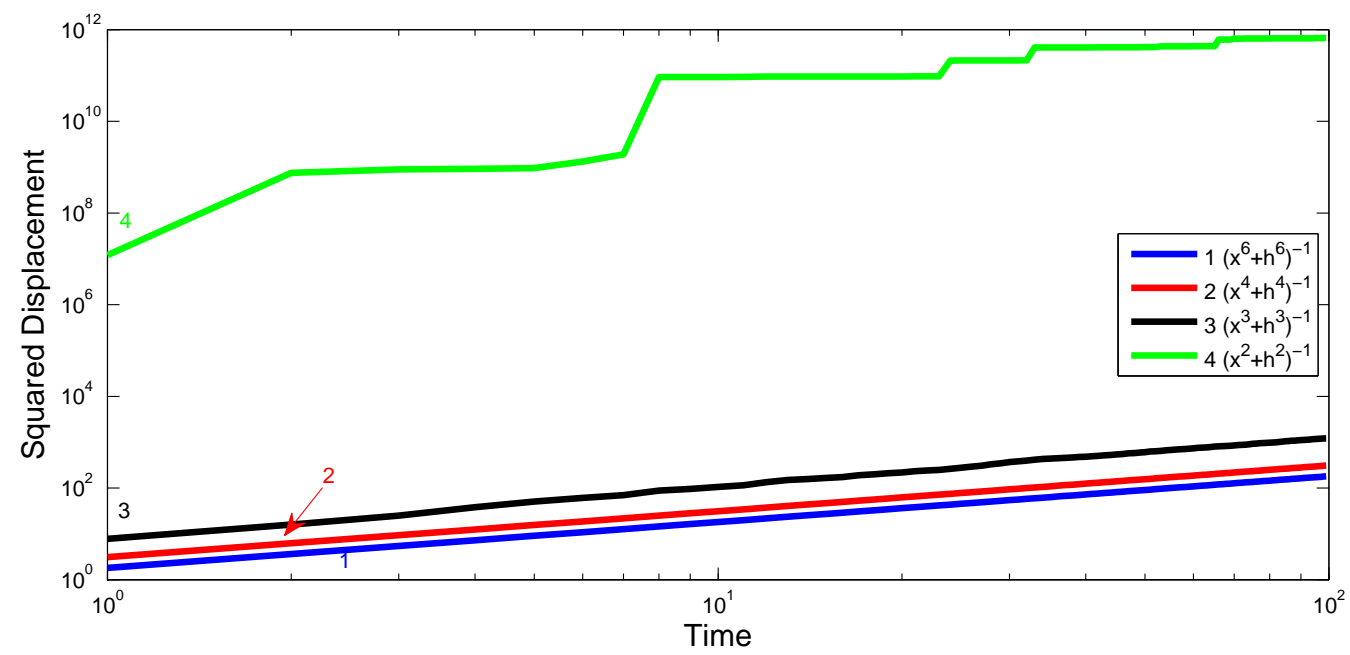

Figure 10. The predicted and estimated mean squared displacement, [13], for various distributions based on $\left(x^{\mu}+h^{\mu}\right)^{-1}$ all with $y=1$ and $C=\frac{1}{2}$. There is no consistency to the direction of movement. The lines for the lower powers, i.e. $\left(x^{3}+h^{3}\right)^{-1}$ and $\left(x^{2}+h^{2}\right)^{-1}$, are estimated by averaging a large number of randomly generated paths, the other lines are produced using the MSD described by Eq. (2.1). 
Interestingly for the case of $C=\frac{9}{10}$ the half-normal distribution is faster than the distribution based on $e^{-a x^{1.5}}$, which is unexpected because the half-normal distribution has a larger power. This is due to the shape of the distributions near the origin and is only the case with value of $C$ close to 1 .

For ease of comparison table 7 shows the fastest distributions for each method of comparison and for three values of $C$. We can clearly see that different methods for comparing distributions result in different orders of magnitudes of displacement. Interestingly the fat-tailed distributions, distributions that lack a variance, are not always the fastest when $C$ has different values. For example when $C=\frac{1}{10}$ the distribution $\frac{a^{3}}{6} e^{-a \sqrt[3]{x}}$ is faster than $2 h^{2}(x+h)^{-3}$ and $\frac{3 \sqrt{3} h^{2}}{2 \pi}\left(x^{3}+h^{3}\right)^{-1}$ both of which lack variances.

Table .1 shows the value of the integral between 0 and $y$ for the general case and the three cases we are focusing on.

Tables .2 and .3 show the parameter, mean distance and square mean distance when the integral is equal to $\frac{9}{10}$ and $\frac{1}{10}$ respectively as in table 5 . While tables .4 and .5 show the variance and order of fastest when the integral is equal to $\frac{9}{10}$ and $\frac{1}{10}$ respectively as in table 6 .

Acknowledgements. This study was partially supported by the PhD Scholarship from the College of Science and Engineering of the University of Leicester (to J.C.).

\section{References}

[1] R.J. Webby, R.G. Webster. Are we ready for pandemic influenza? Science, (2003), 302, 1519-1522.

[2] K.P. Myers, C.W. Olsen, G.C. Gray. Cases of Swine Influenza in Humans: A Review of the Literature. Clinical Infectious Diseases, (2007), 44 (8), 1084-1088.

[3] M. Goeijenbier, J.J. van Kampen, C.B. Reusken, M.P. Koopmans, E.C. van Gorp. Ebola virus disease: a review on epidemiology, symptoms, treatment and pathogenesis. Neth J Med., (2014), 72 (9), 442-448.

[4] S. Bhatt, P.W Gething, O.J. Brady, et al. The global distribution and burden of dengue. Nature, (2013), 496, 504-507.

[5] D. Normile. Surprising new dengue virus throws a spanner in disease control efforts. Science, (2013), $342,415$.

[6] D. Musso, E.J. Nilles, V.M. Cao-Lormeau. Rapid spread of emerging Zika virus in the Pacific area. Clinical Microbiology and Infection, (2014), 20(10), 595-596.

[7] L. Hufnagel, D. Brockmann, T. Geisel. Forecast and control of epidemics in a globalized world. Proc. Natl Acad. Sci. USA, (2004), 101, 15124-15129.

[8] D. Brockmann, L. Hufnagel, T. Geisel. The scaling laws of human travel. Nature, (2006), 439, 462-465.

[9] K. Khan, J. Arino, W. Hu, etc. Spread of a Novel Influenza A (H1N1) Virus via Global Airline Transportation. New Engl J Med, (2009), 361, 212-214.

[10] P. Turchin. Quantitative Analysis of Movement. Sunderland, Sinauer, 1998.

[11] G.M. Viswanathan, M.G.E. da Luz, E.P. Raposo, H.E. Stanley. The Physics of Forag- ing: An Introduction to Random Searches and Biological Encounters. Cambridge UK, Cambridge University Press, 2011.

[12] E.A. Codling, M.J. Plank, S. Benhamou. Random walk models in biology. J. R. Soc. Interface, (2008), 5, 813-834.

[13] P.M. Kareiva, N. Shigesada. Analyzing insect movement as a correlated random walk. Oecologia (Berlin), (1983), 56, 234-238.

[14] R. Brown. A brief account of microscopical observations made in the months of June, July and August, 1827, on the particles contained in the pollen of plants; and the general existence of active molecules in organic and inorganic bodies. Philos. Mag., (1828), 4, 161-173.

[15] A. Einstein. Investigations on the Theory of the Brownian Movement. Dover Publications. [Republication of the original 1926 translation]

[16] H.C. Berg. Random Walks in Biology. Princeton University Press, Princeton, 1983.

[17] V. Mendez, D. Campos, F. Bartumeus. Stochastic foundations in movement ecology: anomalous diffusion, front propagation and random searches. Springer, Berlin, 2014.

[18] H.G. Othmer, S.R. Dunbar,W. Alt. Models of dispersal in biological systems. Math. Biol., (1988), 26, 263-298.

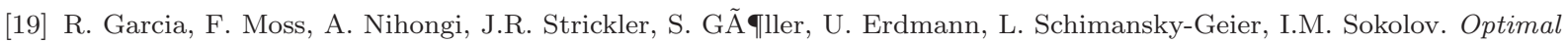
foraging by zooplankton within patches: The case of Daphnia. Mathematical Biosciences, (2007), 207,165-188.

[20] S.V. Petrovskii, N.B. Petrovskaya, D. Bearup. Multiscale approach to pest insect monitoring: random walks, pattern formation, synchronization, and networks. Phys. Life Rev., (2014), 11, 467-525.

[21] J.A. Byers. Correlated random walk equations of animal dispersal resolved by simulation. Ecology, (2001), 82(6), 16801690.

[22] F. Jopp, H. Reuter. Dispersal of carabid beetles - Emergence of distribution patterns. Ecological Modelling, (2005), 186, 389-405.

[23] J.D. Choules. Mathematical models of animal movement. Unpublished MMaths (Hons.) dissertation. University of Leicester, (2015). 


\begin{tabular}{|c|c|c|c|c|c|c|}
\hline Distributions & Equal Variance & Equal Mean & $\begin{array}{c}\text { Equal } \\
\text { Square Mean }\end{array}$ & If $C=\frac{1}{2}$ & If $C=\frac{9}{10}$ & If $C=\frac{1}{10}$ \\
\hline $\begin{array}{l}\frac{\sqrt{2}}{\sigma \sqrt{\pi}} e^{-\frac{x^{2}}{2 \sigma^{2}}} \text { or } \\
\text { Half-Normal }\end{array}$ & 2 nd & 8 th & 2 nd & 11 th & 11 th & 11 th \\
\hline$\frac{a^{\frac{2}{3}}}{\Gamma\left(\frac{5}{3}\right)} e^{-a x^{1.5}}$ & $3 r d$ & 7 th & $3 \mathrm{rd}$ & 10th & 12 th & 9 th \\
\hline $\begin{array}{c}\lambda e^{-\lambda x} \text { or } \\
\text { Exponential }\end{array}$ & 4 th & 5 th & 4 th & 8 th & 10 th & 8 th \\
\hline$\frac{a^{2}}{2} e^{-a \sqrt{x}}$ & 6 th & 4 th & 6 th & 6 th & 7 th & 6 th \\
\hline$\frac{a^{3}}{6} e^{-a \sqrt[3]{x}}$ & 7 th & $3 \mathrm{rd}$ & 7 th & 5 th & 6 th & $3 r d$ \\
\hline $\begin{array}{l}\frac{\mu-1}{h^{1-\mu}}(x+h)^{-\mu} \text { or } \\
\text { Power-Law } \mu>3\end{array}$ & $\begin{array}{c}\text { Between } \\
4 \text { th and 8th }\end{array}$ & $\begin{array}{c}\text { Between } \\
\text { 2nd and 5th }\end{array}$ & $\begin{array}{c}\text { Between } \\
4 \text { th and 8th }\end{array}$ & $\begin{array}{c}\text { Between } \\
4 \text { th and } 13 \text { th }\end{array}$ & $\begin{array}{c}\text { Between } \\
5 \text { th and 13th }\end{array}$ & $\begin{array}{c}\text { Between } \\
5 \text { th and } 13 \text { th }\end{array}$ \\
\hline $\begin{array}{l}2 h^{2}(x+h)^{-3} \text { or } \\
\text { Power-Law } \mu=3\end{array}$ & $\mathrm{~N} / \mathrm{A}$ & $1 \mathrm{st}$ & $\mathrm{N} / \mathrm{A}$ & $3 \mathrm{rd}$ & 4 th & 4 th \\
\hline $\begin{array}{c}h(x+h)^{-2} \text { or } \\
\text { Power-Law } \mu=2\end{array}$ & $\mathrm{~N} / \mathrm{A}$ & $\mathrm{N} / \mathrm{A}$ & $\mathrm{N} / \mathrm{A}$ & $1 \mathrm{st}$ & $1 \mathrm{st}$ & $1 \mathrm{st}$ \\
\hline$\frac{3 h^{5}}{\pi}\left(x^{6}+h^{6}\right)^{-1}$ & $1 \mathrm{st}$ & 9th & $1 \mathrm{st}$ & 12 th & 9th & 12 th \\
\hline$\frac{2^{\frac{3}{2}} h^{3}}{\pi}\left(x^{4}+h^{4}\right)^{-1}$ & 4 th & 5 th & 4 th & 9 th & 8 th & 10th \\
\hline$\frac{3 \sqrt{3} h^{2}}{2 \pi}\left(x^{3}+h^{3}\right)^{-1}$ & $\mathrm{~N} / \mathrm{A}$ & $1 \mathrm{st}$ & $\mathrm{N} / \mathrm{A}$ & 7 th & $3 r d$ & 7 th \\
\hline$\frac{2 h}{\pi}\left(x^{2}+h^{2}\right)^{-1}$ & $\mathrm{~N} / \mathrm{A}$ & $\mathrm{N} / \mathrm{A}$ & $\mathrm{N} / \mathrm{A}$ & $2 n d$ & 2 nd & $2 n d$ \\
\hline
\end{tabular}

TABLE 7. Looking at which is the fastest for different methods of comparison between all the distributions. 


\begin{tabular}{|c|c|c|c|c|}
\hline Distributions & General Case & If $C=\frac{1}{2}$ & If $C=\frac{9}{10}$ & If $C=\frac{1}{10}$ \\
\hline $\begin{array}{l}\frac{\sqrt{2}}{\sigma \sqrt{\pi}} e^{-\frac{x^{2}}{2 \sigma^{2}}} \text { or } \\
\text { Half-Normal }\end{array}$ & $y=\sqrt{2} \sigma \cdot e r f^{-1}(C)$ & $y \approx 0.6745 \sigma$ & $y \approx 1.645 \sigma$ & $y \approx 0.1257 \sigma$ \\
\hline$\frac{a^{\frac{2}{3}}}{\Gamma\left(\frac{5}{3}\right)} e^{-a x^{1.5}}$ & $y=\left(\frac{1}{a} \Gamma^{-1}\left(\frac{2}{3},(1-C)\left(\Gamma\left(\frac{2}{3}\right)\right)\right)\right)^{\frac{2}{3}}$ & $y \approx 0.5215 a^{-\frac{2}{3}}$ & $y \approx 1.420 a^{-\frac{2}{3}}$ & $y \approx 0.0913 a^{-\frac{2}{3}}$ \\
\hline $\begin{array}{c}\lambda e^{-\lambda x} \text { or } \\
\text { Exponential }\end{array}$ & $y=\frac{-1}{\lambda} \ln (1-C)$ & $y \approx 0.6931 \lambda^{-1}$ & $y \approx 2.303 \lambda^{-1}$ & $y \approx 0.1054 \lambda^{-1}$ \\
\hline$\frac{a^{2}}{2} e^{-a \sqrt{x}}$ & $y=\frac{\left(W\left(\frac{C-1}{e}\right)+1\right)^{2}}{a^{2}}$ & $y \approx 2.817 a^{-2}$ & $y \approx 15.13 a^{-2}$ & $y \approx 0.2828 a^{-2}$ \\
\hline$\frac{a^{3}}{6} e^{-a \sqrt[3]{x}}$ & Solved numerically & $y \approx 19.12 a^{-3}$ & $y \approx 150.7 a^{-3}$ & $y \approx 1.338 a^{-3}$ \\
\hline $\begin{array}{l}\frac{\mu-1}{h^{1-\mu}}(x+h)^{-\mu} \text { or } \\
\text { Power-Law } \mu>3\end{array}$ & $y=h\left((1-C)^{\frac{1}{1-\mu}}-1\right)$ & $0<y \approx 0.4142 h$ & $0<y \approx 2.162 h$ & $0<y \approx 0.0541 h$ \\
\hline $\begin{array}{l}2 h^{2}(x+h)^{-3} \text { or } \\
\text { Power-Law } \mu=3\end{array}$ & $y=h\left((1-C)^{-\frac{1}{2}}-1\right)$ & $y \approx 0.4142 h$ & $y \approx 2.162 h$ & $y \approx 0.0541 h$ \\
\hline $\begin{array}{c}h(x+h)^{-2} \text { or } \\
\text { Power-Law } \mu=2\end{array}$ & $y=h\left((1-C)^{-1}-1\right)$ & $y=h$ & $y=9 h$ & $y \approx 0.1111 h$ \\
\hline$\frac{3 h^{5}}{\pi}\left(x^{6}+h^{6}\right)^{-1}$ & Solved numerically & $y \approx 0.5251 h$ & $y \approx 1.089 h$ & $y \approx 0.1047 h$ \\
\hline$\frac{2^{\frac{3}{2}} h^{3}}{\pi}\left(x^{4}+h^{4}\right)^{-1}$ & Solved numerically & $y \approx 0.5664 h$ & $y \approx 1.394 h$ & $y \approx 0.1111 h$ \\
\hline$\frac{3 \sqrt{3} h^{2}}{2 \pi}\left(x^{3}+h^{3}\right)^{-1}$ & Solved numerically & $y \approx 0.6415 h$ & $y \approx 1.985 h$ & $y \approx 0.1210 h$ \\
\hline$\frac{2 h}{\pi}\left(x^{2}+h^{2}\right)^{-1}$ & $y=h \cdot \tan \left(\frac{\pi C}{2}\right)$ & $y=h$ & $y \approx 6.314 h$ & $y \approx 0.1584 h$ \\
\hline
\end{tabular}

TABLE .1. The general case of the integration between 0 and y being equal to a constant $C$ and the cases where $C=\frac{1}{2}, \frac{9}{10}$ and $\frac{1}{10}$ for distributions based on $e^{-a x^{1.5}}, e^{-a \sqrt{x}}, e^{-a \sqrt[3]{x}},\left(x^{4}+h^{4}\right)^{-1},\left(x^{6}+h^{6}\right)^{-1},\left(x^{3}+h^{3}\right)^{-1}$ and $\left(x^{2}+h^{2}\right)^{-1}$ and the half-normal, exponential and standard power-law distributions. 


\begin{tabular}{|c|c|c|c|}
\hline Distributions & $y=1$ and $C=\frac{9}{10}$ & $\begin{array}{c}\text { Mean Distance } \\
-E(x)\end{array}$ & $\begin{array}{c}\text { Mean Squared } \\
\text { Distance - } E\left(x^{2}\right)\end{array}$ \\
\hline $\begin{array}{l}\frac{\sqrt{2}}{\sigma \sqrt{\pi}} e^{-\frac{x^{2}}{2 \sigma^{2}}} \text { or } \\
\text { Half-Normal }\end{array}$ & $\sigma=\left(\sqrt{2} e r f^{-1}\left(\frac{9}{10}\right)\right)^{-1}$ & $\frac{1}{\sqrt{\pi} e r f^{-1}\left(\frac{9}{10}\right)} \approx 0.4851$ & $\frac{1}{2\left(e r f^{-1}\left(\frac{9}{10}\right)\right)^{2}} \approx 0.3696$ \\
\hline$\frac{a^{\frac{2}{3}}}{\Gamma\left(\frac{5}{3}\right)} e^{-a x^{1.5}}$ & $a=\left(\Gamma^{-1}\left(\frac{2}{3}, \frac{1}{10}\left(\Gamma\left(\frac{2}{3}\right)\right)\right)\right)$ & $\begin{array}{c}\frac{2 \Gamma\left(\frac{4}{3}\right)}{3\left(\Gamma^{-1}\left(\frac{2}{3}, \frac{1}{10}\left(\Gamma\left(\frac{2}{3}\right)\right)\right)\right)^{\frac{2}{3}} \Gamma\left(\frac{5}{3}\right)} \\
\approx 0.4643\end{array}$ & $\begin{array}{c}\frac{2}{3\left(\Gamma^{-1}\left(\frac{2}{3}, \frac{1}{10}\left(\Gamma\left(\frac{2}{3}\right)\right)\right)\right)^{\frac{4}{3}} \Gamma\left(\frac{5}{3}\right)} \\
\approx 0.3661\end{array}$ \\
\hline $\begin{array}{c}\lambda e^{-\lambda x} \text { or } \\
\text { Exponential }\end{array}$ & $\lambda=\ln (10)$ & $\frac{1}{\ln (10)} \approx 0.4343$ & $\frac{2}{(\ln (10))^{2}} \approx 0.3772$ \\
\hline$\frac{a^{2}}{2} e^{-a \sqrt{x}}$ & $a=\left(W\left(\frac{-1}{10 e}\right)+1\right)$ & $\frac{6}{\left(W\left(\frac{-1}{10 e}\right)+1\right)^{2}} \approx 0.3966$ & $\frac{120}{\left(W\left(\frac{-1}{10 e}\right)+1\right)^{4}} \approx 0.5242$ \\
\hline$\frac{a^{3}}{6} e^{-a \sqrt[3]{x}}$ & $a \approx 5.322$ & $\approx 0.3980$ & $\approx 0.8872$ \\
\hline $\begin{array}{l}\frac{\mu-1}{h^{1-\mu}}(x+h)^{-\mu} \text { or } \\
\text { Power-Law } \mu>3\end{array}$ & $\begin{aligned} & (\sqrt{10}-1)^{-1}<h \\
= & \left(\left(\frac{1}{10}\right)^{\frac{1}{1-\mu}}-1\right)^{-1}\end{aligned}$ & $\frac{1}{(\mu-2)\left(\left(\frac{1}{10}\right)^{\frac{1}{1-\mu}}-1\right)}<0.4625$ & $\frac{2}{(\mu-3)(\mu-2)\left(\left(\frac{1}{10}\right)^{\frac{1}{1-\mu}}-1\right)^{2}}$ \\
\hline $\begin{array}{c}2 h^{2}(x+h)^{-3} \text { or } \\
\text { Power-Law } \mu=3\end{array}$ & $h=(\sqrt{10}-1)^{-1}$ & $\frac{1}{(\sqrt{10}-1)} \approx 0.4625$ & $\mathrm{~N} / \mathrm{A}$ \\
\hline $\begin{array}{c}h(x+h)^{-2} \text { or } \\
\text { Power-Law } \mu=2\end{array}$ & $h=\frac{1}{9}$ & $\mathrm{~N} / \mathrm{A}$ & $\mathrm{N} / \mathrm{A}$ \\
\hline$\frac{3 h^{5}}{\pi}\left(x^{6}+h^{6}\right)^{-1}$ & $h \approx 0.9185$ & $\approx 0.5303$ & $\approx 0.4218$ \\
\hline$\frac{2^{\frac{3}{2}} h^{3}}{\pi}\left(x^{4}+h^{4}\right)^{-1}$ & $h \approx 0.7174$ & $\approx 0.5073$ & $\approx 0.5146$ \\
\hline$\frac{3 \sqrt{3} h^{2}}{2 \pi}\left(x^{3}+h^{3}\right)^{-1}$ & $h \approx 0.5039$ & $\approx 0.5039$ & $\mathrm{~N} / \mathrm{A}$ \\
\hline$\frac{2 h}{\pi}\left(x^{2}+h^{2}\right)^{-1}$ & $h=0.1584$ & $\mathrm{~N} / \mathrm{A}$ & $\mathrm{N} / \mathrm{A}$ \\
\hline
\end{tabular}

TABLE .2. The case when the integration between 0 and y is equal to a constant, $C=\frac{9}{10}$. We have the values of the parameter, mean distance and square mean distance for distributions based on $e^{-a x^{1.5}}, e^{-a \sqrt{x}}, e^{-a \sqrt[3]{x}},\left(x^{4}+h^{4}\right)^{-1},\left(x^{6}+h^{6}\right)^{-1}$, $\left(x^{3}+h^{3}\right)^{-1}$ and $\left(x^{2}+h^{2}\right)^{-1}$ and the half-normal, exponential and standard power-law distributions. 


\begin{tabular}{|c|c|c|c|}
\hline Distributions & $y=1$ and $C=\frac{1}{10}$ & $\begin{array}{l}\text { Mean Distance } \\
-E(x)\end{array}$ & $\begin{array}{c}\text { Mean Squared } \\
\text { Distance }-E\left(x^{2}\right)\end{array}$ \\
\hline $\begin{array}{l}\frac{\sqrt{2}}{\sigma \sqrt{\pi}} e^{-\frac{x^{2}}{2 \sigma^{2}}} \text { or } \\
\text { Half-Normal }\end{array}$ & $\sigma=\left(\sqrt{2} e r f^{-1}\left(\frac{1}{10}\right)\right)^{-1}$ & $\frac{1}{\sqrt{\pi} e r f^{-1}\left(\frac{1}{10}\right)} \approx 6.350$ & $\frac{1}{2\left(e r f^{-1}\left(\frac{1}{10}\right)\right)^{2}} \approx 63.33$ \\
\hline$\frac{a^{\frac{2}{3}}}{\Gamma\left(\frac{5}{3}\right)} e^{-a x^{1.5}}$ & $a=\left(\Gamma^{-1}\left(\frac{2}{3}, \frac{9}{10}\left(\Gamma\left(\frac{2}{3}\right)\right)\right)\right)$ & $\begin{array}{c}\frac{2 \Gamma\left(\frac{4}{3}\right)}{3\left(\Gamma^{-1}\left(\frac{2}{3}, \frac{9}{10}\left(\Gamma\left(\frac{2}{3}\right)\right)\right)\right)^{\frac{2}{3}} \Gamma\left(\frac{5}{3}\right)} \\
\approx 7.225\end{array}$ & $\begin{array}{c}\frac{2}{3\left(\Gamma^{-1}\left(\frac{2}{3}, \frac{9}{10}\left(\Gamma\left(\frac{2}{3}\right)\right)\right)\right)^{\frac{4}{3}} \Gamma\left(\frac{5}{3}\right)} \\
\approx 88.65\end{array}$ \\
\hline $\begin{array}{c}\lambda e^{-\lambda x} \text { or } \\
\text { Exponential }\end{array}$ & $\lambda=\ln \left(\frac{10}{9}\right)$ & $\frac{1}{\ln \left(\frac{10}{9}\right)} \approx 9.491$ & $\frac{2}{\left(\ln \left(\frac{10}{9}\right)\right)^{2}} \approx 180.2$ \\
\hline$\frac{a^{2}}{2} e^{-a \sqrt{x}}$ & $a=\left(W\left(\frac{-9}{10 e}\right)+1\right)$ & $\frac{6}{\left(W\left(\frac{-9}{10 e}\right)+1\right)^{2}} \approx 21.21$ & $\frac{120}{\left(W\left(\frac{-9}{10 e}\right)+1\right)^{4}} \approx 1500$ \\
\hline$\frac{a^{3}}{6} e^{-a \sqrt[3]{x}}$ & $a \approx 1.102$ & $\approx 44.83$ & $\approx 11260$ \\
\hline $\begin{array}{l}\frac{\mu-1}{h^{1-\mu}}(x+h)^{-\mu} \text { or } \\
\text { Power-Law } \mu>3\end{array}$ & $\begin{aligned} & \left(\sqrt{\frac{10}{9}}-1\right)^{-1}<h \\
= & \left(\left(\frac{9}{10}\right)^{\frac{1}{1-\mu}}-1\right)^{-1}\end{aligned}$ & $\frac{1}{(\mu-2)\left(\left(\frac{9}{10}\right)^{\frac{1}{1-\mu}}-1\right)}<18.49$ & $\frac{2}{(\mu-3)(\mu-2)\left(\left(\frac{9}{10}\right)^{\frac{1}{1-\mu}}-1\right)^{2}}$ \\
\hline $\begin{array}{c}2 h^{2}(x+h)^{-3} \text { or } \\
\text { Power-Law } \mu=3\end{array}$ & $h=\left(\sqrt{\frac{10}{9}}-1\right)^{-1}$ & $\frac{1}{\left(\sqrt{\frac{10}{9}}-1\right)} \approx 18.49$ & $\mathrm{~N} / \mathrm{A}$ \\
\hline $\begin{array}{c}h(x+h)^{-2} \text { or } \\
\text { Power-Law } \mu=2\end{array}$ & $h=9$ & $\mathrm{~N} / \mathrm{A}$ & $\mathrm{N} / \mathrm{A}$ \\
\hline$\frac{3 h^{5}}{\pi}\left(x^{6}+h^{6}\right)^{-1}$ & $h \approx 9.550$ & $\approx 5.514$ & $\approx 45.60$ \\
\hline$\frac{2^{\frac{3}{2}} h^{3}}{\pi}\left(x^{4}+h^{4}\right)^{-1}$ & $h \approx 9.004$ & $\approx 6.367$ & $\approx 81.06$ \\
\hline$\frac{3 \sqrt{3} h^{2}}{2 \pi}\left(x^{3}+h^{3}\right)^{-1}$ & $h \approx 8.266$ & $\approx 8.266$ & $\mathrm{~N} / \mathrm{A}$ \\
\hline$\frac{2 h}{\pi}\left(x^{2}+h^{2}\right)^{-1}$ & $h=6.314$ & $\mathrm{~N} / \mathrm{A}$ & $\mathrm{N} / \mathrm{A}$ \\
\hline
\end{tabular}

TABLE .3. The case when the integration between 0 and y is equal to a constant, $C=\frac{1}{10}$. We have the values of the parameter, mean distance and square mean distance for distributions based on $e^{-a x^{1.5}}, e^{-a \sqrt{x}}, e^{-a \sqrt[3]{x}},\left(x^{4}+h^{4}\right)^{-1},\left(x^{6}+h^{6}\right)^{-1}$, $\left(x^{3}+h^{3}\right)^{-1}$ and $\left(x^{2}+h^{2}\right)^{-1}$ and the half-normal, exponential and standard power-law distributions. 


\begin{tabular}{|c|c|c|c|}
\hline Distributions & $y=1$ and $C=\frac{9}{10}$ & Variance & Fastest \\
\hline $\begin{array}{l}\frac{\sqrt{2}}{\sigma \sqrt{\pi}} e^{-\frac{x^{2}}{2 \sigma^{2}}} \text { or } \\
\text { Half-Normal }\end{array}$ & $\sigma=\left(\sqrt{2} e r f^{-1}\left(\frac{9}{10}\right)\right)^{-1}$ & $\frac{\pi-2}{2 \pi\left(e r f^{-1}\left(\frac{9}{10}\right)\right)^{2}} \approx 0.1343$ & 11 th \\
\hline$\frac{a^{\frac{2}{3}}}{\Gamma\left(\frac{5}{3}\right)} e^{-a x^{1.5}}$ & $a=\left(\Gamma^{-1}\left(\frac{2}{3}, \frac{1}{10}\left(\Gamma\left(\frac{2}{3}\right)\right)\right)\right)$ & $\begin{array}{c}\frac{6 \Gamma\left(\frac{5}{3}\right)-2 \Gamma\left(\frac{4}{3}\right)}{9\left(\Gamma^{-1}\left(\frac{2}{3}, \frac{1}{10}\left(\Gamma\left(\frac{2}{3}\right)\right)\right)\right)^{\frac{4}{3}}\left(\Gamma\left(\frac{5}{3}\right)\right)^{2}} \\
\approx 0.1505\end{array}$ & 12 th \\
\hline $\begin{array}{c}\lambda e^{-\lambda x} \text { or } \\
\text { Exponential }\end{array}$ & $\lambda=\ln (10)$ & $\frac{1}{(\ln (10))^{2}} \approx 0.1886$ & 10 th \\
\hline$\frac{a^{2}}{2} e^{-a \sqrt{x}}$ & $a=\left(W\left(\frac{-1}{10 e}\right)+1\right)$ & $\frac{84}{\left(W\left(\frac{-1}{10 e}\right)+1\right)^{4}} \approx 0.3669$ & 7 th \\
\hline$\frac{a^{3}}{6} e^{-a \sqrt[3]{x}}$ & $a \approx 5.322$ & $\approx 0.7288$ & 6 th \\
\hline $\begin{array}{l}\frac{\mu-1}{h^{1-\mu}}(x+h)^{-\mu} \text { or } \\
\text { Power-Law } \mu>3\end{array}$ & $\begin{aligned} & (\sqrt{10}-1)^{-1}<h \\
= & \left(\left(\frac{1}{10}\right)^{\frac{1}{1-\mu}}-1\right)^{-1}\end{aligned}$ & $\frac{\mu-1}{(\mu-3)(\mu-2)^{2}\left(\left(\frac{1}{10}\right)^{\frac{1}{1-\mu}}-1\right)^{2}}$ & $\begin{array}{c}\text { Between } \\
\text { 5th and 13th }\end{array}$ \\
\hline $\begin{array}{c}2 h^{2}(x+h)^{-3} \text { or } \\
\text { Power-Law } \mu=3\end{array}$ & $h=(\sqrt{10}-1)^{-1}$ & $\mathrm{~N} / \mathrm{A}$ & 4 th \\
\hline $\begin{array}{c}h(x+h)^{-2} \text { or } \\
\text { Power-Law } \mu=2\end{array}$ & $h=\frac{1}{9}$ & $\mathrm{~N} / \mathrm{A}$ & $1 \mathrm{st}$ \\
\hline$\frac{3 h^{5}}{\pi}\left(x^{6}+h^{6}\right)^{-1}$ & $h \approx 0.9185$ & $\approx 0.1406$ & 9 th \\
\hline$\frac{2^{\frac{3}{2}} h^{3}}{\pi}\left(x^{4}+h^{4}\right)^{-1}$ & $h \approx 0.7174$ & $\approx 0.2573$ & 8th \\
\hline$\frac{3 \sqrt{3} h^{2}}{2 \pi}\left(x^{3}+h^{3}\right)^{-1}$ & $h \approx 0.5039$ & $\mathrm{~N} / \mathrm{A}$ & 3rd \\
\hline$\frac{2 h}{\pi}\left(x^{2}+h^{2}\right)^{-1}$ & $h=0.1584$ & $\mathrm{~N} / \mathrm{A}$ & 2 nd \\
\hline
\end{tabular}

TABLE .4. The case when the integration between 0 and y is equal to a constant, $C=\frac{9}{10}$. We have the values of the parameter and variance for distributions based on $e^{-a x^{1.5}}, e^{-a \sqrt{x}}, e^{-a \sqrt[3]{x}},\left(x^{4}+h^{4}\right)^{-1},\left(x^{6}+h^{6}\right)^{-1},\left(x^{3}+h^{3}\right)^{-1}$ and $\left(x^{2}+h^{2}\right)^{-1}$ and the half-normal, exponential and standard power-law distributions. 


\begin{tabular}{|c|c|c|c|}
\hline Distributions & $y=1$ and $C=\frac{1}{10}$ & Variance & Fastest \\
\hline $\begin{array}{l}\frac{\sqrt{2}}{\sigma \sqrt{\pi}} e^{-\frac{x^{2}}{2 \sigma^{2}}} \text { or } \\
\text { Half-Normal }\end{array}$ & $\sigma=\left(\sqrt{2} e r f^{-1}\left(\frac{1}{10}\right)\right)^{-1}$ & $\frac{\pi-2}{2 \pi\left(e r f^{-1}\left(\frac{1}{10}\right)\right)^{2}} \approx 23.01$ & 11 th \\
\hline$\frac{a^{\frac{2}{3}}}{\Gamma\left(\frac{5}{3}\right)} e^{-a x^{1.5}}$ & $a=\left(\Gamma^{-1}\left(\frac{2}{3}, \frac{9}{10}\left(\Gamma\left(\frac{2}{3}\right)\right)\right)\right)$ & $\begin{array}{c}\frac{6 \Gamma\left(\frac{5}{3}\right)-2 \Gamma\left(\frac{4}{3}\right)}{9\left(\Gamma^{-1}\left(\frac{2}{3}, \frac{9}{10}\left(\Gamma\left(\frac{2}{3}\right)\right)\right)\right)^{\frac{4}{3}}\left(\Gamma\left(\frac{5}{3}\right)\right)^{2}} \\
\approx 36.44\end{array}$ & 9 th \\
\hline $\begin{array}{c}\lambda e^{-\lambda x} \text { or } \\
\text { Exponential }\end{array}$ & $\lambda=\ln \left(\frac{10}{9}\right)$ & $\frac{1}{\left(\ln \left(\frac{10}{9}\right)\right)^{2}} \approx 90.08$ & 8th \\
\hline$\frac{a^{2}}{2} e^{-a \sqrt{x}}$ & $a=\left(W\left(\frac{-9}{10 e}\right)+1\right)$ & $\frac{84}{\left(W\left(\frac{-9}{10 e}\right)+1\right)^{4}} \approx 1050$ & 6 th \\
\hline$\frac{a^{3}}{6} e^{-a \sqrt[3]{x}}$ & $a \approx 1.102$ & $\approx 9246$ & $3 r d$ \\
\hline $\begin{array}{l}\frac{\mu-1}{h^{1-\mu}}(x+h)^{-\mu} \text { or } \\
\text { Power-Law } \mu>3\end{array}$ & $\begin{aligned} & \left(\sqrt{\frac{10}{9}}-1\right)^{-1}<h \\
= & \left(\left(\frac{9}{10}\right)^{\frac{1}{1-\mu}}-1\right)^{-1}\end{aligned}$ & $\frac{\mu-1}{(\mu-3)(\mu-2)^{2}\left(\left(\frac{9}{10}\right)^{\frac{1}{1-\mu}}-1\right)^{2}}$ & $\begin{array}{c}\text { Between } \\
5 \text { th and 13th }\end{array}$ \\
\hline $\begin{array}{c}2 h^{2}(x+h)^{-3} \text { or } \\
\text { Power-Law } \mu=3\end{array}$ & $h=\left(\sqrt{\frac{10}{9}}-1\right)^{-1}$ & $\mathrm{~N} / \mathrm{A}$ & 4 th \\
\hline $\begin{array}{c}h(x+h)^{-2} \text { or } \\
\text { Power-Law } \mu=2\end{array}$ & $h=9$ & $\mathrm{~N} / \mathrm{A}$ & $1 \mathrm{st}$ \\
\hline$\frac{3 h^{5}}{\pi}\left(x^{6}+h^{6}\right)^{-1}$ & $h \approx 9.550$ & $\approx 15.20$ & 12 th \\
\hline$\frac{2^{\frac{3}{2}} h^{3}}{\pi}\left(x^{4}+h^{4}\right)^{-1}$ & $h \approx 9.004$ & $\approx 40.53$ & 10th \\
\hline$\frac{3 \sqrt{3} h^{2}}{2 \pi}\left(x^{3}+h^{3}\right)^{-1}$ & $h \approx 8.266$ & $\mathrm{~N} / \mathrm{A}$ & 7 th \\
\hline$\frac{2 h}{\pi}\left(x^{2}+h^{2}\right)^{-1}$ & $h=6.314$ & $\mathrm{~N} / \mathrm{A}$ & 2nd \\
\hline
\end{tabular}

TABLE .5. The case when the integration between 0 and y is equal to a constant, $C=\frac{1}{10}$. We have the values of the parameter and variance for distributions based on $e^{-a x^{1.5}}, e^{-a \sqrt{x}}, e^{-a \sqrt[3]{x}},\left(x^{4}+h^{4}\right)^{-1},\left(x^{6}+h^{6}\right)^{-1},\left(x^{3}+h^{3}\right)^{-1}$ and $\left(x^{2}+h^{2}\right)^{-1}$ and the half-normal, exponential and standard power-law distributions. 
[24] M.F. Shlesinger, G.M. Zaslavsky, J. Klafter. Strange kinetics. Nature, (1993), 363, 31-37.

[25] D.W. Sims, E.J. Southall, N.E. Humphries, G.C. Hays, C.J.A. Bradshaw, J.W. Pitchford, A. James, M.Z. Ahmed, A.S. Brierly, M.A. Hindell, D. Morritt, M.K. Musyl, D. Righton, E.L.C. Shepard, V.J. Wearmouth, R.P. Wilson, M.J. Witt, J.D. Metcalfe, Scaling laws of marine predator search behaviour. Nature, (2008), 451, 1098-1102.

[26] G.M. Viswanathan, V. Afanasyev, S.V. Buldyrev, E.J. Murphy, P.A. Prince, H. E. Stanley Lévy flight search patterns of wandering albatrosses. Nature, (1996), 381, 413-415.

[27] G. Ramos-Fernández, J.L. Mateos, O. Miramontes, G. Cocho, H. Larralde, B. Ayala-Orozco. Lévy walk patterns in the foraging movements of spider monkeys (Ateles geoffroyi). Behav. Ecol. Sociobiol., (2004), 55, 223-230.

[28] F. Bartumeus, E.P. Raposo, M.G.E. da Luz, G.M. Viswanathan. Stochastic optimal foraging theory. In: Dispersal, Individual Movement, and Spatial Ecology: A Mathematical Perspective. (Lewis, M.A., Maini, P., Petrovskii, S.V., Eds.) Springer Lecture Notes in Mathematics Vol. 2071. Springer, Berlin, 2013.

[29] B. Mandelbrot. The Fractal Geometry of Nature. Freeman, New York, 1977.

[30] A.M. Reynolds, A.D. Smith, D.R. Reynolds, N.L. Carreck, J.L. Osborne. oneybees perform optimal scale-free searching flights when attempting to locate a food source. J. Exp. Biol., (2007), 210, 3763-3770.

[31] R. Kawai, S.V. Petrovskii. Multi-scale properties of random walk models of animal movement: lessons from statistical inference. Proc. R. Soc. A, (2012), 468, 1428-1451.

[32] D. Bearup, C.M. Benefer, S.V. Petrovskii, R. Blackshaw. Revisiting Brownian motion as a description of animal movement: a comparison to experimental movement data. Methods in Ecology and Evolution, (2016), 7, 1525-1537.

[33] L.A.D. Rodrigues, D.C. Mistro, E.R. Cara, N. Petrovskaya, S.V. Petrovskii. Patchy invasion of stage-structured alien species with short-distance and long-distance dispersal. Bull. Math. Biol., (2015), 77, 1583-1619.

[34] B.O. Koopman. On Distributions Admitting a Sufficient Statistic. Transactions of the American Mathematical Society, (1936), 39(3), 399-409.

[35] E.J.G. Pitman. Sufficient statistics and intrinsic accuracy. Mathematical Proceedings of the Cambridge Philosophical Society, (1936), 32, 567-579.

[36] D.A.S. Fraser. On sufficiency and the Exponential Family. Journal of the Royal Statistical Society. Series B (Methodological), (1963), 25(1), 115-123.

[37] E.A. Codling, J.W. Pitchford, S.D. Simpson. Group navigation and the "many wrongs principles" in models of animal movement. Ecology, (2007), 88, 1864-1870.

[38] S. Benhamou. How many animals really do the Levy walk? Ecology, (2007), 88, 1962-1969.

[39] A. James, M.J. Plank. On fitting power laws to ecological data. (2007), arXiv:0712.0613 [q-bio.QM]. 